\title{
Sunitinib treatment promotes metastasis of drug- resistant renal cell carcinoma via TFE3 signaling pathway
}

\author{
Luchao Li', Shuo Zhao', Zhengfang Liu', Nianzhao Zhang', Shuo Pang', Jikai Liu', Cheng Liu and Yidong Fan ${ }^{1}$
}

\begin{abstract}
Receptor tyrosine kinase (RTK) inhibitors, such as sunitinib and sorafenib, remain the first-line drugs for the treatment of mRCC. Acquired drug resistance and metastasis are the main causes of treatment failure. However, in the case of metastasis Renal Cell Cancer (mRCC), which showed a good response to sunitinib, we found that long-term treatment with sunitinib could promote lysosome biosynthesis and exocytosis, thereby triggering the metastasis of RCC. By constructing sunitinib-resistant cell lines in vivo, we confirmed that TFE3 plays a key role in the acquired resistance to sunitinib in RCC. Under the stimulation of sunitinib, TFE3 continued to enter the nucleus, promoting the expression of endoplasmic reticulum (ER) protein E-Syt1. E-Syt1 and the lysosomal membrane protein Syt7 form a heterodimer, which induces ER fragmentation, $\mathrm{Ca}_{2}{ }^{+}$release, and lysosomal exocytosis. Lysosomal exocytosis has two functions: pumping sunitinib out from the cytoplasm, which promotes resistance to sunitinib in RCC, releasing cathepsin $B$ (CTSB) into the extracellular matrix (ECM), which can degrade the ECM to enhance the invasion and metastasis ability of RCC. Our study found that although sunitinib is an effective drug for the treatment of mRCC, once RCC has acquired resistance to sunitinib, sunitinib treatment will promote metastasis.
\end{abstract}

\section{Introduction}

Renal cell carcinoma (RCC) accounts for approximately $2 \%$ of all adult malignancies worldwide ${ }^{1}$. Clear cell renal cell carcinoma (ccRCC), the most common histologic subtype of $\mathrm{RCC}^{2}$, is characterized by a loss of the von Hippel-Lindau (VHL) gene with induction of hypoxiainducible factor (HIF) and vascular endothelium growth factor $(\mathrm{VEGF})^{3-6}$. Radical nephrectomy can enable patients with early-stage RCC to obtain a very impressive 5 -year survival rate $(92.6 \%)^{7}$. However, over $20 \%$ of patients are primarily diagnosed at the metastatic stage, and over $30 \%$ suffer metastasis after surgery ${ }^{8}$. Antiangiogenic receptor tyrosine kinase inhibitors (TKIs) such as sunitinib and sorafenib remain the first-line treatment

\footnotetext{
Correspondence: Jikai Liu (14111270004@fudan.edu.cn) or Yidong Fan (fanyd@sdu.edu.cn)

'Department of Urology, Qilu Hospital, Shandong University, Jinan, China 2Department of Urology, Peking University Third Hospital, Beijing, China Edited by S. Tait
}

for mRCC. Unfortunately, most patients eventually develop resistance to TKI treatment. For the clinical management of mRCC patients, a more definite understanding of the mechanisms of tumor metastasis and TKI resistance is urgently needed. Lysosomes are intracellular vesicles bound by phospholipid bilayer membranes. Their biological functions include clearance of dysfunctional organelles, degraded proteins, and phagocytosis ${ }^{9-13}$. The main lysosomal acid hydrolases, cathepsins (CTS), are involved in tumor progression 0tumor metastasis is the degradation of the extracellular matrix (ECM) by matrix metalloproteinases such as MMP9. The exocytosis of cathepsin B into the ECM via lysosomes can trigger MMP-9 activation so as to degrade the ECM and dissolute cell-cell adhesion molecules to promote cancer metastasis $^{14,15}$. In addition, as a kind of hydrophobic weak base synthetic drug, sunitinib can be trapped and sequestered in acidic lysosomes so as to induce sunitinib resistance in ccRCC $^{16-18}$. The coordinated lysosomal expression and

\section{(c) The Author(s) 2021}

(c) (i) Open Access This article is licensed under a Creative Commons Attribution 4.0 International License, which permits use, sharing, adaptation, distribution and reproduction cc) in any medium or format, as long as you give appropriate credit to the original author(s) and the source, provide a link to the Creative Commons license, and indicate if changes were made. The images or other third party material in this article are included in the article's Creative Commons license, unless indicated otherwise in a credit line to the material. If material is not included in the article's Creative Commons license and your intended use is not permitted by statutory regulation or exceeds the permitted use, you will need to obtain permission directly from the copyright holder. To view a copy of this license, visit http://creativecommons.org/licenses/by/4.0/. 
regulation (CLEAR) gene network is the upstream regulator of lysosome biogenesis. Transcription factor EB (TFEB) and transcription factor E3 (TFE3) are two core activators of the CLEAR pathway and depend on mitochondrial translational initiation factor (MITF) family members ${ }^{19-21}$.

We treated a mRCC patient with sunitinib. After 4 years of continuous sunitinib treatment, the patient developed new metastasis to the cervical lymph nodes. Tandem mass tag (TMT) proteomic sequencing was used to analyze metastatic RCC lesions compared with sunitinib-sensitive RCC lesions. Surprisingly, we found that the lysosome enzyme pathway was strongly enriched in the upregulated proteins, and the ECM receptor and cell adhesion pathways were enriched among the downregulated proteins. Based on the patient's medication history, it is likely that the amplification of the lysosomal pathway is caused by TFEB/TFE3 translocation into the nucleus, thereby activating the CLEAR gene regulation network. We further infer that the new metastasis in the patient is most likely due to the fact that after drug resistance in the primary lesion, sustained TKI drugs still inhibited the VEGF-mTOR pathway, causing TFE3/TFEB to lose the restriction of phosphorylation and thus enter the nucleus. In other words, if the treatment strategy is not changed after primary lesion resistance to TKI, continuous TKI treatment will result in the activation of the TFEB/TFE3lysosome pathway to trigger RCC metastasis.

\section{Materials and methods The ITRAQ quantitative proteomics Protein extraction and labeling}

Samples were obtained with the consent of the ethics committee of Qilu hospital and the patient herself. After treated with $1 \mathrm{ml}$ of lysate containing $7 \mathrm{~m}$ urea, $2 \mathrm{~m}$ thiourea, and $0.1 \%$ chaps, tissues from each group were homogenized by $\mathrm{TiO}_{2}$ redox beads $(70 \mathrm{~Hz}, 120 \mathrm{~s}$ at $5000 \times g \times 5 \mathrm{~min}$ at $4^{\circ} \mathrm{C}$ ). Collect the supernatant with centrifugal force of $15000 \times g$ at $4{ }^{\circ} \mathrm{C}$ for $15 \mathrm{~min}$. The protein concentration was determined according to the manufacturer's protocol (Qinglian Biotech Co., Ltd, Beijing, China). In total $200 \mu \mathrm{g}$ samples were digested overnight with $4 \mu \mathrm{g}$ trypsin at $37^{\circ} \mathrm{C}$. labeling with incubating $200 \mu \mathrm{g}$ protein with a reducing agent $(5 \mu \mathrm{l}, 200 \mathrm{~mm})$ at $55^{\circ} \mathrm{C}$ for $60 \mathrm{~min}$, then adding iodoacetamide $(5 \mu \mathrm{l}$, $375 \mathrm{mM}$ ) for $10 \mathrm{~min}$ without light at RT. Next, adding dissolution buffer (200 $\mu \mathrm{l} 100 \mathrm{~mm}$ Absciex), and centrifuged at $15000 \times g$ for $15 \mathrm{~min}$. Digesting each sample with trypsin $(4 \mu \mathrm{g})$ overnight at RT. Then lyophilizing all the samples and dissolving with dissolution buffer $(100 \mathrm{mM})$. Labeling samples according to the protocol of TMT kit (PN: 90064, Thermo Scientific, Waltham, MA USA).

\section{Identification of peptide}

Resuspending the peptide by buffer A (0.1\% FA, 2\% $\mathrm{ACN}, 20 \mu \mathrm{l})$ and collecting the supernatant with centrifugal force of $12000 \mathrm{rpm}$ for $10 \mathrm{~min}$. Then a nano UPLC-MS/MS system which was composed of a Nanoflow HPLC system (EASY-nLC1000 system, Thermo Scientific) and Q-Exactive mass spectrometer (Thermo Scientific) was used to identify. The database is NCBI Mus musculus_20160428 and Proteome Discoverer 1.4 is used for data processing.

\section{Protein identification and quantification}

Parameters for identifications: precursor ion mass tolerance, $\pm 15 \mathrm{ppm}$; fragmentation mass tolerance, $\pm 20 \mathrm{mmu}$; max missed cleavages,2; static modification, carboxyamidomethylation (57.021 Da) of Cys residues; dynamic modifications, oxidation modification $(+15.995 \mathrm{Da})$ of Met residues $p \leq 0.05$ and difference ratio $\geq 1.2$ were chosen for bioinformatics analysis.

\section{Cell culture}

The HeLa cell line and $786 \mathrm{O}$ cell line were obtained from the Chinese Academy of Science Cell Bank (Shanghai, China). The media of culturing $786 \mathrm{O}, 786 \mathrm{O} /$ OE, 786 O/OE-SR cells were RPMI-1640 supplemented with $10 \%$ fetal bovine serum, 100 units $/ \mathrm{ml}$ penicillin, and streptomycin (Gibco Invitrogen, Monza, Italy). In total 293 T was cultured in Dulbecco's modified Eagle's medium (DMEM, Gibco Invitrogen) with 10\% FBS, 100 units/ $\mathrm{ml}$ penicillin, and streptomycin. All cells were maintained under the condition of $37^{\circ} \mathrm{C}$ with $5 \% \mathrm{CO}_{2}$. All the cell lines were recently authenticated STR without mycoplasma contamination.

\section{Reagents and antibodies}

Sunitinib (s7781), DC661(S8808), MG132(S2619), 3MA(S2767), CA-074ME(S7420), Ionomycin (s7074), Puromycin(s7417) were purchased from Selleckchem (Houston, TX, USA). Fluo-3(46393), Earle's Balanced Salt Solution (EBSS, E7510) were purchased from SigmaAldrich. The primary antibodies against E-Syt1 (ab118805), FTH1(ab231253), TFE3 (ab196681), CTSB (ab125067), Syt7(121383), mCherry (ab183628), GAPDH (ab8245), LC3B (ab192890), GFP (ab290), HA (ab9110), mTOR (ab32028), p-mTOR (ab109268), MMP9 (ab76003), H3 (ab1791) were purchased from Abcam (Cambridge, Cambridgeshire, UK). The primary antibody of FAM14B (21573-1-AP) were purchased from Proteintech (Chicago, IL, USA). The horseradish peroxidase (HRP) labeled Goat anti-Rabbit (ab6721) and Rabbit antiMouse (ab6728) secondary antibodies were purchases from Abcam. 


\section{Cell viability assay}

In total $3 \times 10^{\wedge} 3$ cells/well were seeded into 96-well plates overnight. Then the cells were treated with new complete media containing sunitinib with different concentration for $48 \mathrm{~h}$. After that, each well was added $10 \mu \mathrm{L}$ Cell Counting Kit-8 (CCK-8) solution (Dojindo, Kumamoto, Japan) and the plates were incubated at $37^{\circ} \mathrm{C}$ for $1 \mathrm{~h}$. Finally, a microplate reader was used to test the absorbance at $450 \mathrm{~nm}$. Three separated repeats were performed.

\section{Wound healing assay}

The indicated cells were plated in six-well plates to test the migration capability. Drawing a line shape in the wells of the 6 -well by using a $100-\mu \mathrm{L}$ pipette tip. Then replace the medium with $2 \mathrm{~mL}$ of serum-free culture medium containing different concentration of sunitinib. Scratches were pictured at $0 \mathrm{~h}$ and $24 \mathrm{~h}$ after the medium was replaced immediately after. Changes in the scratches were imaged after $24 \mathrm{~h}$. The cells migration capability was judged based on variations in the widths of scratches. Three separated repeats were performed.

\section{Transwell assay}

Before seeding $2 \times 10^{\wedge}$ cells $(200 \mu \mathrm{L}$ serum-free RPMI1640 medium) into the champers (Millipore, Billerica, MA, USA), the upper facial of membrane $(8 \mu \mathrm{m}$ porefilters) was coated with the $50 \mu \mathrm{L}$ matrigel $(200 \mathrm{ng} / \mathrm{mL}$, BD Biosciences, NJ, USA) and RPMI-1640 with 10\% FBS was added to the lower chambers. Then the seeding cells were incubated $12 \mathrm{~h}$ for migration and $24 \mathrm{~h}$ for invasion at $37^{\circ} \mathrm{C}$. Cells retained on the upper facial of the membrane were gently wiped off and cells migrated to the bottom of the membrane were fixed with $4 \%$ paraformaldehyde, stained by crystal violet solution for $20 \mathrm{~min}$. Visualization and statistics under 100 times magnification. Experiments were repeated three times with similar results.

\section{Enzyme linked immunosorbent assay (ELISA)}

ELISA for node mouse serum Ferritin (ab157713, Abcam) was performed according to the manufacturer's instructions.

Small interfering RNA (siRNA), plasmid, lentiviral synthesis Small interference RNA (siRNA) targeting E-Syt1, TFE3, short hairpin RNA (shRNA) targeting E-Syt1, CTSB, FAM134B, Syt7, TFE3, overexpressing plasmids driving TFE3, E-Syt1, E-Syt1-GFP, FAM134B-mCherry, Sty7, Syt-GFP were synthesized by Genepharma (Shanghai, China). The lentivirus containing TFE3 overexpressing plasmid, sh-TFE3 plasmid, sh-Syt7 plasmid, EATR system, CCER system were synthesized by Shanghai Genechem Co., Ltd. Sty7-mCherry overexpressing plasmid and corresponding mutation plasmids were designed by Genepharma (Shanghai, China).

\section{Mass spectrum}

A total of 786 O/OE-SR cells transfected with Esyt1GFP or Syt-GFP plasmid, subjected to incubation with anti-GFP antibody. The proteins having interaction with Esyt1-GFP or Syt7-GFP were pulled down by Co-IP according to Kit' protocols from TaKaRa (TaKaRa BIO, Dalian, China) and performed with MS analysis. SDSPAGE gel was used to separate the peptide. We minced the gel and collected it in a test tube and dried it naturally. The peptide segments in the gel fragments were reduced by $10 \mathrm{mM}$ DTT at $56^{\circ} \mathrm{C}$ for $30 \mathrm{~min}$, and then alkylated with $50 \mathrm{mM}$ IAM for $30 \mathrm{~min}$ in the dark. Samples were digested by trypsin for $14 \mathrm{~h}$ at $37^{\circ} \mathrm{C}$. The samples were acidified by formic acid and desalted by $\mathrm{C} 18$ cartridge. Then samples were dried by vacuum centrifugation. The Orbitrap Q Exactive HF-X mass spectrometer (Thermo Fisher) was used to test samples.

\section{Immunohistochemistry}

The clinical patient' tumor tissues and xenograft tumor tissues were fixed in $10 \%$ formalin for $48 \mathrm{~h}$. After dehydration and embedment, the tissues were made into paraffin sections. Then xylene and a series of descending dilutions of ethanol were used to deparaffinize. We used a microwave oven to perform antigen retrieval by heating to boiling in citrate antigen retrieval solution for $15 \mathrm{~min}$. The activity of endogenous peroxidase was blocked by $0.3 \%$ Hydrogen Peroxide at room temperature for $20 \mathrm{~min}$. The slides were then tested with the antibody of TFE3 respectively $(1: 200)$ at $4{ }^{\circ} \mathrm{C}$ overnight and then the sections were tested with HRP-streptavidin-conjugated secondary antibody at room temperature for $30 \mathrm{~min}$. DAB and hematoxylin staining were used to develop the expression of TFE3 in tissues. The research protocol conducted was approved by the ethics committee of Shandong University on 26 January 2016 (protocol number: A9180112).

\section{EATR assay}

The principle of EATR is as follows: when ER-phagy is not triggered, the tandem fluorescent proteins are stable in the cytoplasm, When ER-phagy occurs with lysosomes engulfing these two fluorescent proteins mediated by RAMP4, the green fluorescence will quench by the instability of GFP in acid lysosomes so as to leave red fluorescence from mCherry ${ }^{22}$.

\section{Luciferase report assay}

TFE3 overexpression plasmid, pGL3 basic empty, ESyt1 overexpression plasmid, E-Syt1 mutated plasmid, Syt7 overexpression plasmid, Syt7 mutated plasmid, FTH1 overexpression plasmid, FTH1 mutated plasmid were constructed by Genepharma (Shanghai, China). $293 \mathrm{~T}$ cells $\left(8 \times 10^{4}\right.$ cells per well $)$ were seeded into 24 well 
a

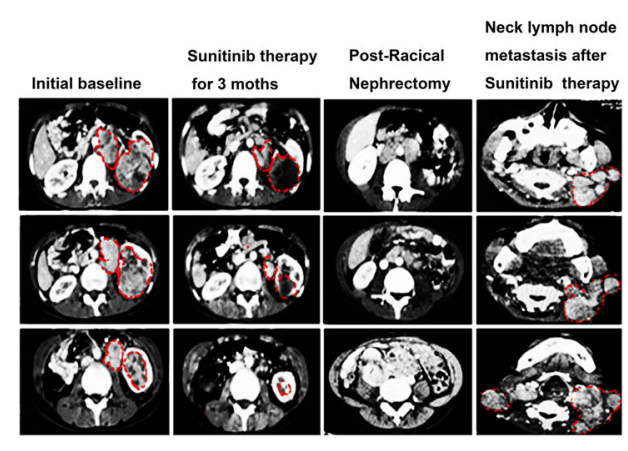

C

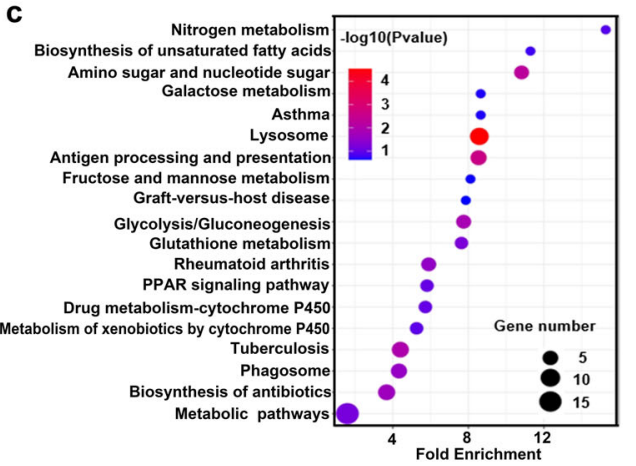

e

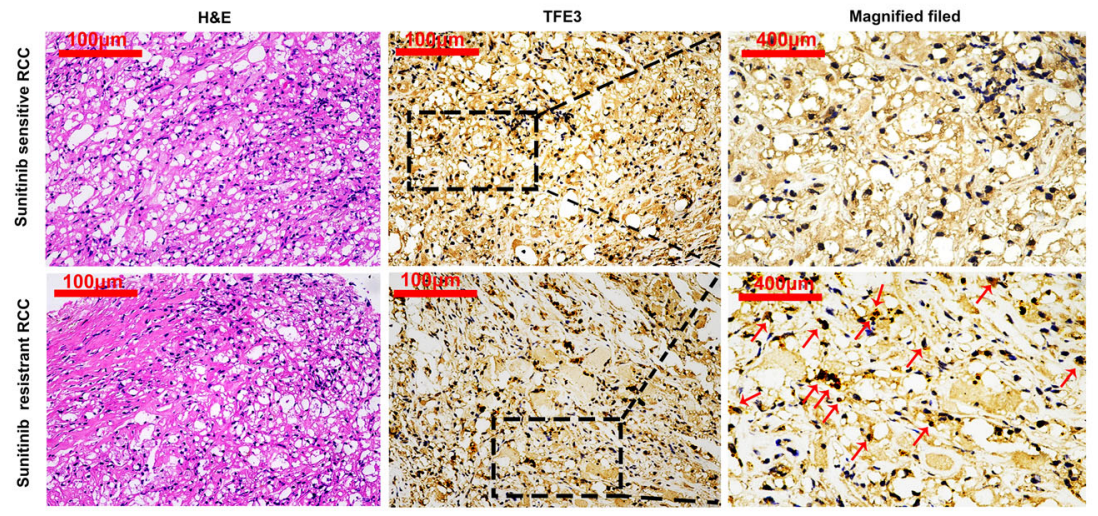

f b

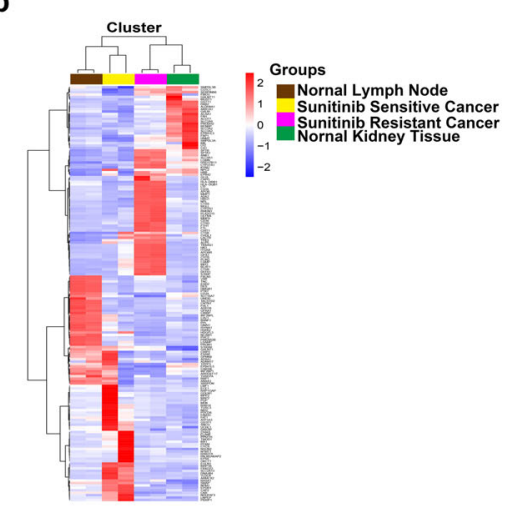

d

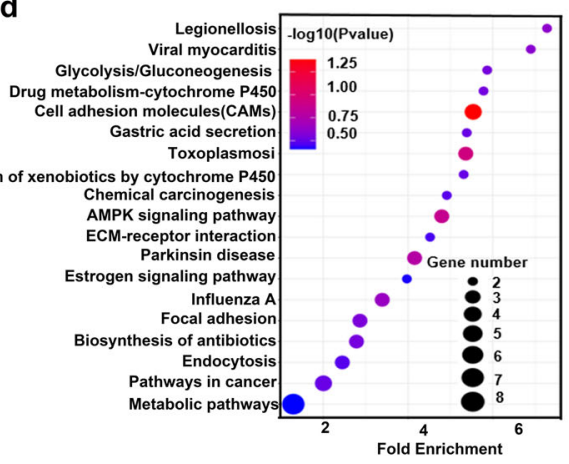

Magnified filed

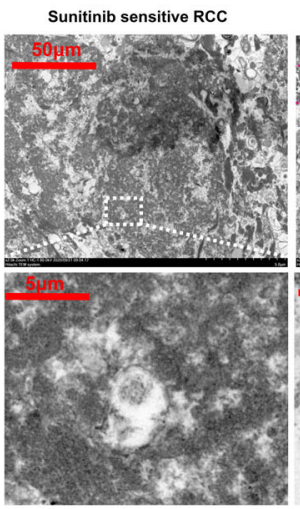

Sunitinib resistrant RCC

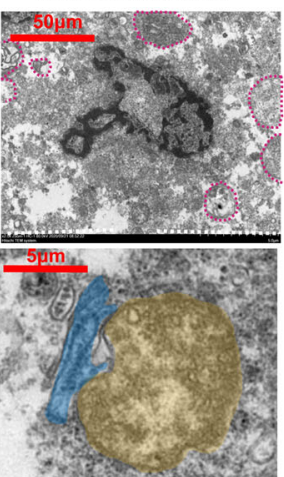

Fig. 1 (See legend on next page.) 
(see figure on previous page)

Fig. 1 Translocation of TFE3 promoted metastasis of sunitinib-resistant RCC by mediating lysosome synthesis. a CT scans of different disease stages from a ccRCC patient: sunitinib sensitive stage, one month after radical nephrectomy, new metastatic lesions occurred in left lymph node by 4 years' sunitinib treatment (from left to right). b Heat map of TMT proteomics from cancer tissues from different disease stages. $\mathbf{c}$ GO functional annotation analyzed the up-regulated proteins and the most differentially expressed proteins were enriched in the lysosome. $\mathbf{d}$ GO functional annotation analyzed the down-regulated proteins and the most differentially expressed proteins were enriched in CAMS, ECM receptor interaction, and focal adhesion. e Representative images of hematoxylin-eosin (H\&E) staining and TFE3 staining by immunohistochemical (IHC) in sunitinib sensitive tissues and sunitinib resistant tissues. Scale bar, $100 \mu \mathrm{m}$ and $400 \mu \mathrm{m}$. f Representative TEM images of lysosome (red dotted circle) in sunitinib sensitive tissues and sunitinib resistant tissues Scale bar $50 \mu \mathrm{m}$. Higher magnification insets showed the autophagosome (yellow) was engulfing the ER fragment (blue) in sunitinib-resistant tissues compared with sunitinib-sensitive tissues. Scale bar $5 \mu \mathrm{m}$.

culture plate with $500 \mu \mathrm{l}$ DMEM medium containing $10 \%$ FBS and cultured at $37^{\circ} \mathrm{C}$. On the second day, each group was transfected with indicated plasmids respectively. by transfection reagent Lipofectamine 3000 Reagent (Thermo Fisher). Then $48 \mathrm{~h}$ later, the double luciferase reporter assay system (Promega, Beijing, China) was performed by manufacturer's instructions.

\section{Correlation analysis}

The preprocessed and standardized expression value matrix data (series_matrix document) were downloaded from two GEO datasets and read by $\mathrm{R}$ package (3.6.1) limma package. For different probes mapped to the same gene, we take the mean value of different probes as the final expression value of the gene. The ENSEMBL ID of RNA -seq data of TCGA data (https://www.gencodegenes.org /) is reannotated with GTF annotation file in the instrument to obtain the corresponding gene symbol. The expression profiles of TFE3 and E-Syt1, Syt7, and FTH1 were extracted. Based on Pearson algorithm, nine expression value correlation graphs were drawn through R-package ggplot2.

\section{Transmission electron microscopy}

Cells transfected with Syt7 or clinical tumor specimens (storage at $-80^{\circ} \mathrm{C}$ ) were fixed with $2.5 \%$ glutaraldehyde overnight at $4{ }^{\circ} \mathrm{C}$. After fixation with $1 \%$ osmium tetroxide for $1 \mathrm{~h}$, we dehydrated all the samples with different concentrations of ethanol. Araldite was the embedding reagent. Ultrathin sections of $(50 \mathrm{~nm})$ were stained by uranyl 16 acetate and lead citrate. A cm-120 electron microscope (TECNAI 17 sprite biotwin, Fei, Thermo Scientific) was used to scan the slices.

\section{Quantitative PCR (qPCR)}

RNeasy Mini Kit (Qiagen, Hilden, Germany) was used to extract total RNA. from tissues or cells. Reverse transcription of total RNA was processed by kit instructions from kit (Clontech, Mountain View CA). Quantitative PCR reactions were processed by Fast qPCR Mix SYBR Green Master Mix (TaKaRa, RR430S).

FTH1 forward, 5'-CAACAGTGCTTGGACGGAAC-3' FTH1 reverse, 5'-GAGTCCTGGTGGTAGTTCTGG- ${ }^{\prime}$ CTSB forward, 5'-CTCTTTCCATCCCCTGTCGG-3'
CTSB reverse, 5'-TAGCCACCATTACAGCCGTC-3' Syt7 forward, 5'-GTCAGCCTTAGCGTCACTGT-3' Syt7 reverse, $5^{\prime}$-GACTCATCGTGGGGTGTCTG-3' E-Syt1 forward, 5'-GGTTGCTGGTGCTGATACCT-3' E-Syt1 reverse, 5'-GGCAGGTAGCTCTCGATGAC-3' Lamp1 forward, 5'-CAACACGTTACAGCGTCCAG-3' Lamp1 reverse, 5'-CCTGGGTGCCACTAACACAT-3' GAPDH forward, 5'-A AGCTCACTGGCATGGCCTT- ${ }^{\prime}$ GAPDH reverse, $5^{\prime}$-C TCTCTTCCTCTTGTGCTCT TG-3'.

\section{Nuclear/cytoplasmic protein fractionation}

Nuclear and cytoplasmic fractionation was performed according to The Kit protocols from Abcam (ab113474). After been centrifuged, the nuclear and the cytosolic fraction were collected respectively. Equal volumes of the nuclear and cytoplasmic lysates were tested by Western blot.

\section{Western blot and Co-immunoprecipitation}

After lysing and extracting the total cell protein with RIPA buffer (Beyotime, Shanghai, China), we used BCA Protein Assay Kit (Beyotime, Shanghai, China) to determine the protein concentration. The protein samples were separated by $12 \%$ sodium dodecyl sulphate polyacrylamide gels electrophoresis (SDS-PAGE) $(30 \mu \mathrm{g}$ per lane) and then transferred onto the PVDF membrane (Millipore, Billerica, MA, USA). In total 5\% skimmed milk in TBST $(0.1 \%$ Tween-20 in PBS) was used to block the membrane for $1 \mathrm{~h}$ at room temperature, all the primary antibodies needed were used to incubate the membrane overnight at $4{ }^{\circ} \mathrm{C}$. After washing 3 times by TBST, the bands were then incubated in secondary antibody for $1 \mathrm{~h}$ at room temperature. The enhanced chemiluminescence (ECL) reaction was detected by ChemiDoc XRS + system (Bio-Rad, Hercules, CA). Immunoblot image intensities were tested by Image software. Cells collected from $10 \mathrm{~cm}$ dishes were prepared for immunoprecipitation and performed according to the Kit' protocols from TaKaRa (635721).

\section{Live-cell image}

After transfected with indicated plasmid, $786 \mathrm{O} / \mathrm{OE}-\mathrm{SR}$ cells $\left(5 \times 10^{\wedge}\right)$ were seeded in FluoroDish cell culture 
a

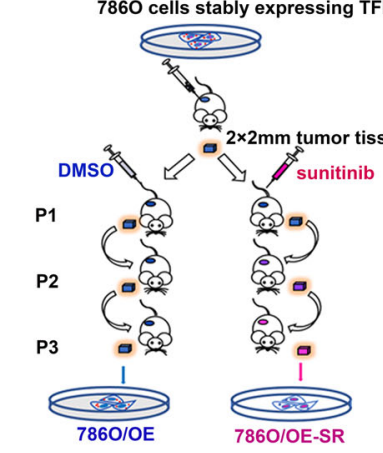

d

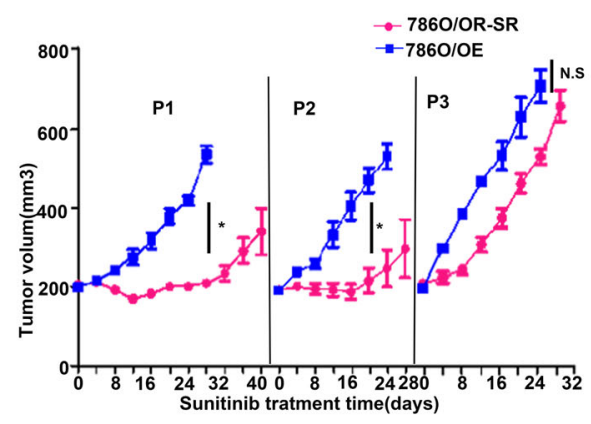

f

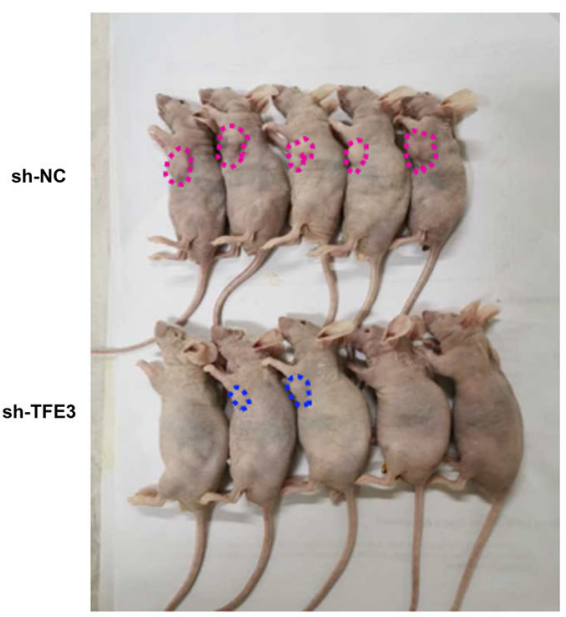

h

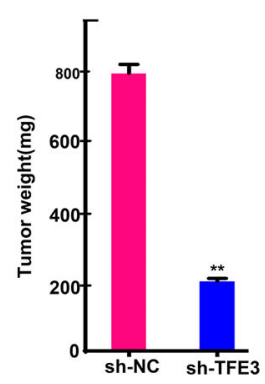

c
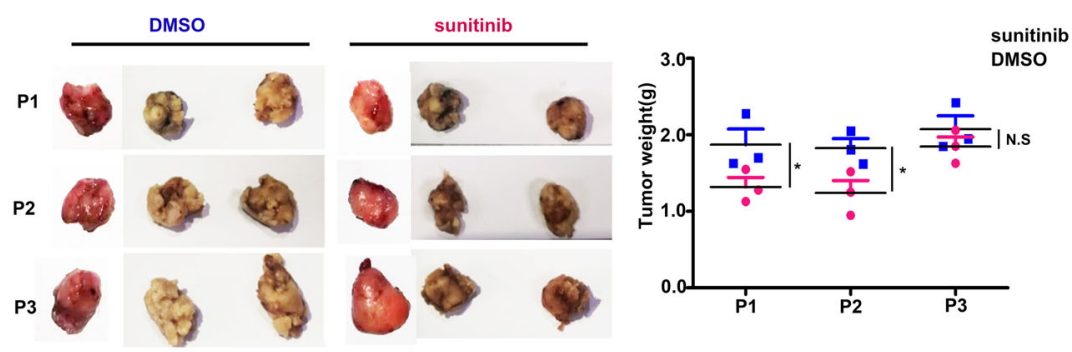

e

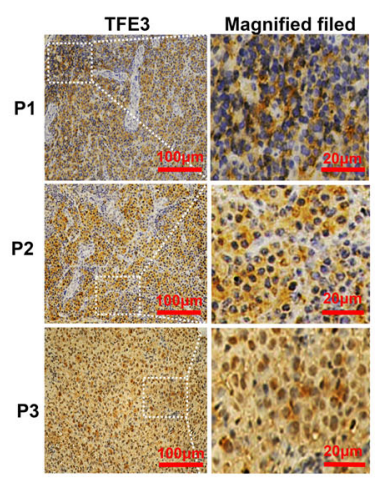

g

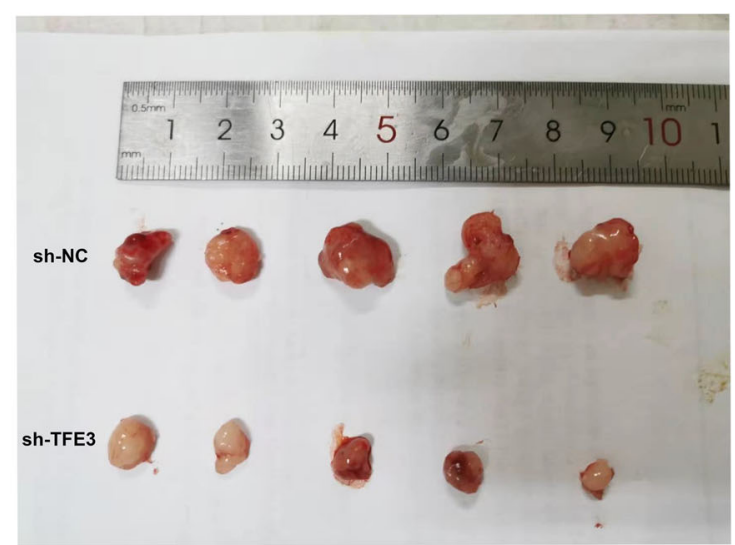

i

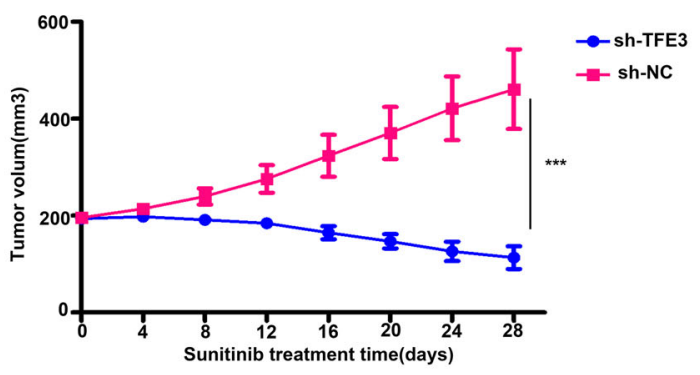

Fig. 2 (See legend on next page.) 
(see figure on previous page)

Fig. 2 TFE3 is the core regulator of sunitinib resistance in vivo. a Describe the process of establishing two cell lines. Subcutaneous tumorigenesis in mice using $786 \mathrm{O}$ cell lines stably expressing TFE3. Control group received DMSO (blue) treatment and experimental group received sunitinib (yellow) treatment. After three generations of treatment, the primary cells were extracted named $786 \mathrm{O} / \mathrm{OE}$ and $786 \mathrm{O} / \mathrm{OE}-\mathrm{SR}$ correspondingly. $\mathbf{b}$, $\mathbf{c}$ Weight of tumor xenografts in mice of every generation (two-tailed Student's $t$ test and two ways ANOVA, ${ }^{*} P<0.05$, NS: not significant). d Tumor volume of every generation (two-tailed Student's $t$ test and two ways ANOVA, ${ }^{*} P<0.05$, NS: not significant). e Representative images of IHC staining TFE3 in P1, P2, P3 xenografts Scale bar, $100 \mu \mathrm{m}$, indicated region $20 \mu \mathrm{m}$. f, g BALB/c nude mice were implanted with 786 O/OE-SR cells which were transfected with lentivirus containing sh-TFE3 or sh-empty vector ( $n=5$ per group) and all mice received sunitinib ( $15 \mathrm{mg} / \mathrm{kg} / \mathrm{day})$ treatment for 30 days. Representative photos of tumors were illustrated. $\mathbf{h}$, i Tumor volumes and weights were lower in mice with TFE3 knockdown xenotransplant. ${ }^{* *} P<0.01,{ }^{* * *} P<0.001$.

dishes (Shengyou Hangzhou China). After the culture medium was removed, post-transfected cells were washed with preheated PBS and counter stained with Hoechst 33342 for 10 min followed by washing in preheated PBS and incubated in preheated culture medium (Life technologies). Then we took the real-time imagines by confocal microscopy (Carl Zeiss Germany). Similarly for ER tracker (Serve Life Science, Shanghai China) staining, after $100 \mu \mathrm{l}$ working solution incubated with indicated cells for $10 \mathrm{~min}$, post transfected cells were washed with preheated PBS and stained with Hoechst 33342 for 10 min followed by washing in preheated PBS and incubated in preheated culture medium (Life technologies).

\section{Detection of intracellular $\mathrm{Ca}^{2+}$ concentration}

After cells received indicated treatment, they were seeded in 6 well plates $\left(5 \times 10^{5}\right.$ well for $14 \mathrm{~h}$. Then cells were incubated with Fluo-3-AM $(0.5 \mu \mathrm{m} /$ well $)$ at $37^{\circ} \mathrm{C}$ for $10 \mathrm{~min}$. Washing 3 times with PBS. The cells were then, and $\mathrm{Ca}^{2+}$ concentration was measured by flow cytometry analysis (BD Biosciences).

\section{Subcutaneous xenograft model and lung metastasis model}

The female BALB/c nude mice ( 3 weeks of age) were purchased in batches from Beijing VitalRiver Laboratory Animal Technology Co., Ltd. All animal work procedures were approved by the Ethics Committee of the Shandong University Qilu Hospital. The 786 O cells stably expressing TFE3 were randomly injected subcutaneously into six nude mice $(5 \times 106$ cells in $200 \mu \mathrm{l} \mathrm{PBS}, n=3$ /group) at 4 weeks of age. Tumor volume was daily measured. Calculate formula of tumor Volume: (large diameter) $\times(\text { small diameter })^{2} / 2$. When the tumor volume approached about $200 \mathrm{~mm}^{3}$, the six tumors bearing mice were randomly assigned to two groups. Randomly one group received sunitinib treatment by tail injection $(15 \mathrm{mg} / \mathrm{kg} /$ day $)$ for 40 days and the other group received equivalent DMSO by tail injection for 24 days as P1 generation. One tumor from each group was cut into small pieces $(2 \times 2 \mathrm{~mm})$ and implanted subsequently to the P2 generation of new nude mice $(n=3$ / group/generation). When the tumor volume approached about $200 \mathrm{~mm}^{3}$, continuous sunitinib treatment (32 days) and DMSO treatment (24 days) were processed in P2 generation mouse. The establishment method of P3 generation is the same as above. The biggest tumor from each group in the P3 generation was selected for primary cell extraction and culture. The primary cells received DMSO treatment is named $786 \mathrm{O} / \mathrm{OE}$ and the primary cells received sunitinib treatment is named $786 \mathrm{O} / \mathrm{OE}-\mathrm{SR}$.

The pro-metastatic activities of Syt7 were tested by the mouse cancer lung metastasis model. $786 \mathrm{O} / \mathrm{OE}-\mathrm{SR}$ cells were infected with lentivirus containing sh-Syt7 plasmid or empty vector plasmid as control and each group contained 5 nude mice. Infected cells were selected with $2 \mu \mathrm{g} /$ $\mathrm{ml}$ puromycin for 1week. Syt7 knockdown cells and control cells $\left(2 \times 10^{5}\right)$ were suspended in $200 \mu \mathrm{L}$ of PBS and implanted by tail vein injection. Randomly each group of mice were treated with sunitinib $(10 \mathrm{mg} / \mathrm{kg} / \mathrm{day})$ or DMSO for 45 days. The mice lungs metastasis was assessed by counting the number of nodules by staining the lung slides with Hematoxylin-eosin (H\&E) staining.

\section{Statistical analysis}

The data of three independent experiments were presented as means \pm the standard deviation (sd). Student's ttest was performed statistical comparisons by using of the results were done using GraphPad Prism 5 software. $P$ value $<0.05$ is considered as statistically significant and are indicated as follows: ${ }^{*} P<0.05$; ${ }^{* * *} P<0.01$; ${ }^{* * * *} P<0.001$.

\section{Result \\ Lysosomal pathway was reactively activated in $\mathrm{mRCC}$ after acquired sunitinib resistance}

A 24-year-old female diagnosed with mRCC using enhanced computed tomography (CT) scan in 2011 (Fig. 1a). In response to sunitinib therapy for 3 months, the tumor size had decreased significantly as shown in CT scan (Fig. 1a). We performed radical nephrectomy of the left kidney for this patient, and the pathological report was ccRCC (pT4N1M1) (Fig. 1e). This patient received postoperative maintenance with sunitinib, and regular followsups were performed to monitor the progress of the disease. After sunitinib therapy for 4 years, this case developed left cervical lymph node metastasis (Fig. 1a). We firmly believed that the tumor samples from the left kidney are sunitinib sensitive, and respectively the samples from lymph nodes are 
a
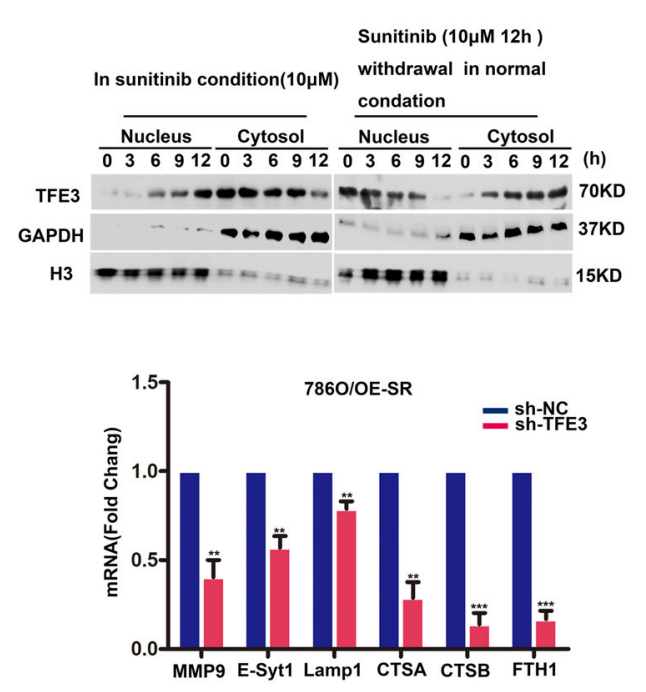

b

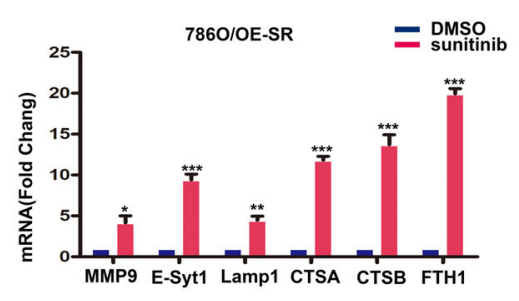

d

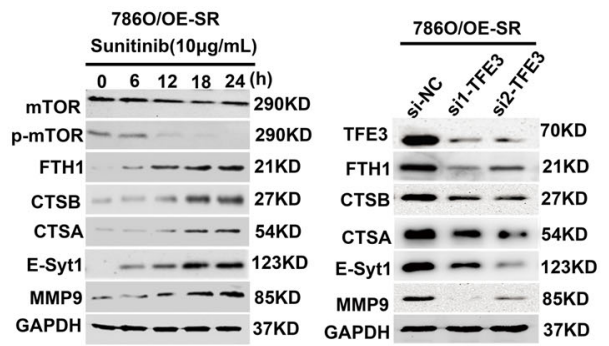

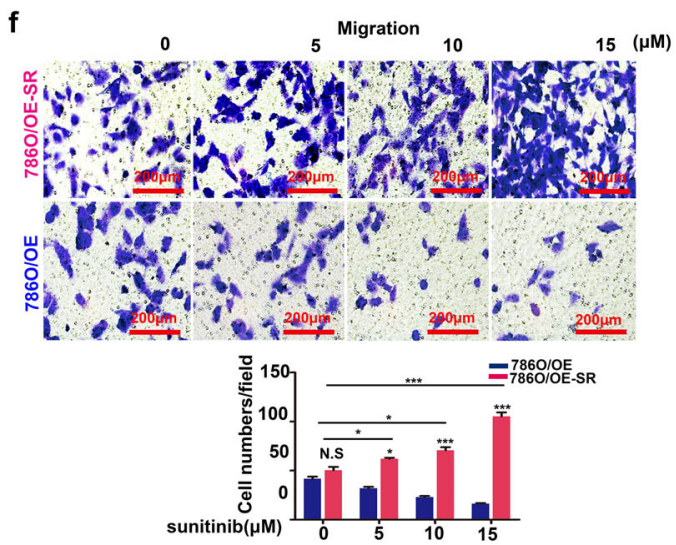

h

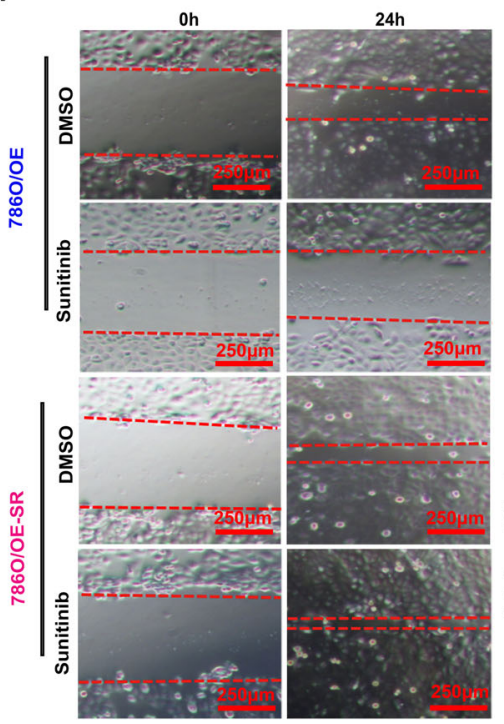

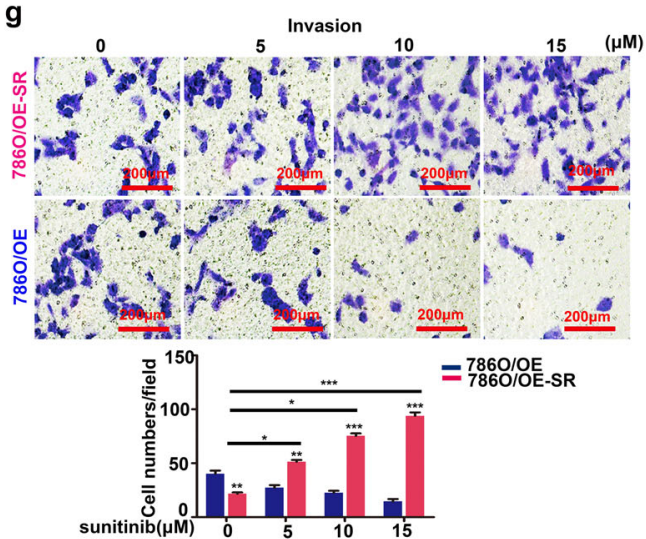

i
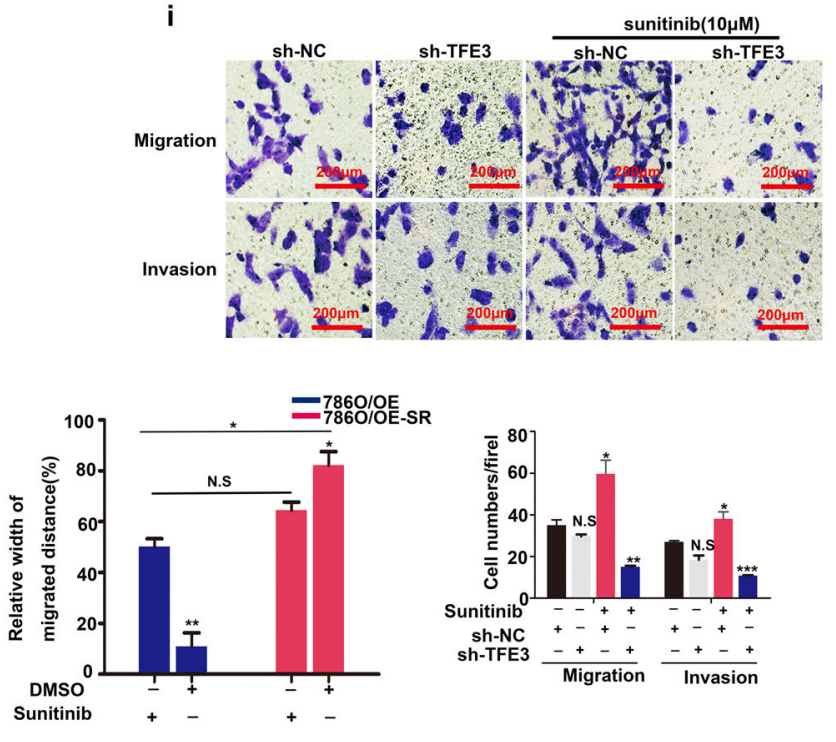

Fig. 3 (See legend on next page.) 
(see figure on previous page)

Fig. 3 Sunitinib stimulated the metastasis of 7860/OE-SR cells by activating TFE3 translocation. a Western blot showed that expression and distribution of TFE3 was dynamic between the nucleus and the cytoplasm. GAPDH and Histone served as specific internal reference markers for cytoplasm and nuclei respectively. $\mathbf{b}$ The target genes of TFE3 were verified in786O/OE-SR cells treated with DMSO or sunitinib (10 $\mu \mathrm{M})$ for $12 \mathrm{~h}$. Data are shown as means \pm SD. ${ }^{*} P<0.05,{ }^{* *} P<0.01,{ }^{* *} P<0.001$, NS: not significant. Data were obtained from three independent experiments. $\mathbf{c}$ After knocking down of TFE3, qPCR verified the target gene of TFE3 in 786 O/OE-SR cells treated with DMSO, sunitinib $(10 \mu \mathrm{M})$ for $12 \mathrm{~h}$. ${ }^{* *} P<0.01,{ }^{* * *} P<$ 0.001. Data are shown as means \pm SD $(n=3)$. $\mathbf{d}$ Proteins downstream of TFE3 in $786 \mathrm{O} / \mathrm{OE}-\mathrm{SR}$ cell line were upregulated with the increase in sunitinib concentration. e After knocking down of TFE3, 786 O/OE-SR were treated with sunitinib $(10 \mu \mathrm{M})$ for $12 \mathrm{~h}$ cells and proteins downstream of TFE3 were down regulated. $\mathbf{f}$ Transwell assay to assess migration after $12 \mathrm{~h}$ incubation of $786 \mathrm{O} / \mathrm{OE}-\mathrm{SR}$ with different concentration of sunitinib compared with 786 O/OE cells. Scale bar, $200 \mu \mathrm{m},{ }^{* * *} P<0.001,{ }^{* *} P<0.01,{ }^{*} P<0.05$, NS: not significant. Data are shown as means \pm SD $(n=3)$. $\mathbf{g}$ Transwell assay to assess invasion after $12 \mathrm{~h}$ incubation of $786 \mathrm{O} / \mathrm{OE}-\mathrm{SR}$ with different concentration of sunitinib compared with $786 \mathrm{O} / \mathrm{OE}$ cells. Scale bar, $200 \mu \mathrm{m}$. ${ }^{* *} P<$ $0.01,{ }^{* * *} P<0.001$. Data are shown as means $\pm \mathrm{SD}(n=3)$. $\mathbf{h}$ Wound healing assays for $786 \mathrm{O} / \mathrm{OE}$ and $786 \mathrm{O} / \mathrm{OE}-\mathrm{SR}$ cells with DMSO, sunitinib $(10 \mu \mathrm{M})$ for $24 \mathrm{~h}$. Scale bar, $250 \mu \mathrm{m}$. ${ }^{*} P<0.05$, ${ }^{*} P<0.01$, NS: not significant. Data are shown as means \pm SD $(n=3)$. i Rescue experiments showed that knockout of TFE3 could reverse the effect of sunitinib on tumor metastasis in 786 O/OE-SR cells. Scale bar, $200 \mu m,{ }^{*} P<0.05,{ }^{* *} P<0.01, N S$ : not significant. Data are shown as means \pm SD $(n=3)$.

sunitinib resistant. We applied TMT Proteomics to analysis the sunitinib sensitive samples and paired lymph metastasis samples. Two sites were randomly selected for each lesion. The heat map analysis was illustrated in Fig. 1b. We analyzed the survival of the proteins with the most obvious difference by the TCGA database (Supplementary Fig. 1). Then bubble chart showed that lysosomal proteins were the most significantly up-regulated protein in metastasis sample compared to sunitinib sensitive tumor sample (Fig. 1c). Correspondingly, the down-expressed proteins were enriched in extracellular matrix, such as cell adhesion molecules (CAMs) and ECM-receptor interaction proteins (Fig. 1d). TFE3 was strongly expressed in the nucleus in the metastatic RCC tissue, and diffused in the cytoplasm in sunitinib sensitive tissue which theoretically consistent with the fact that sunitinib promoted TFE3 translocated into the nucleus by inhibiting VEGF-mTOR signaling pathway (Fig. 1e). There were more and bigger lysosomes in metastasis RCC samples than in sunitinib sensitive RCC samples (Fig. 1f). Interestingly, we surprisingly found abundant ER-phagy phenomena by transmission electron microscope (TEM) (Fig. 1f).

\section{TFE3 induced acquired sunitinib resistance and metastasis} in RCC

In order to confirm the hypothesis that sunitinib can induce metastasis of RCC after drug resistance, we first established two cell line models named $786 \mathrm{O} / \mathrm{OE}$ and 786 O/OE-SR via in vivo experiments (Fig. 2a). By measuring the tumor volume and weight, we can see that the P1 and the P2 xenograft tumors had a fair response to sunitinib, and the P3 xenografts have already showed stable sunitinib resistance and the tumor volume slightly increased with the effect of the drug (Fig. 2b-d). IHC assay showed that in P1 and P2 generation, TFE3 was diffusely distributed in the cytoplasm, but in P3 generation, TFE3 was strongly expressed in the nucleus (Fig. 2e). TFE3 knockdown in $786 \mathrm{O} / \mathrm{OE}-\mathrm{SR}$ cells significantly reduced the growth of tumor xenografts and can reverse the resistance to sunitinib in mice models (Fig. 2f-i)

\section{TFE3 is a core regulator of RCC metastasis induced by long-term sunitinib treatment in vitro}

$786 \mathrm{O} / \mathrm{OE}-\mathrm{SR}$ cells showed stronger sunitinib resistance than 786 O/OE cells and 786 O cells (Supplementary Fig. $2 \mathrm{a})$. TFE3 is restrained in the cytosol under normal conditions and rapidly translocated into the nucleus when in stress conditions ${ }^{21}$. WB experiments confirmed that the basal amount of TFE3 in the nucleus of $786 \mathrm{O} / \mathrm{OE}-\mathrm{SR}$ cells was much more abundant than that in $786 \mathrm{O} / \mathrm{OE}$ cells (Supplementary Fig. 2b). Under normal conditions, TFE3 is restrained in cytosol, but rapidly translocates to the nucleus when cells suffer medium stress condition ${ }^{21}$. WB assay confirmed that the subcellular distribution of TFE3 mediated by sunitinib was a dynamic process. The expression of TFE3 in the nucleus increased in sunitinib condition. After drug withdrawal, TFE3 gradually transferred back to cytoplasm (Fig. 3a). From the TMT Proteomics data, we found that Ferritin and E-Syt1 are dramatically upregulated in mRCC tissue. By WB and qPCR assay, we confirmed that some targets filtered out from proteomics were the downstream proteins of TFE3 in $786 \mathrm{O} / \mathrm{OE}-\mathrm{SR}$ cells (Fig. 3b-e). We also compared these targets at the mRNA and protein levels in $786 \mathrm{O} / \mathrm{OE}$ and 786 O/OE-SR cells (Supplementary Fig. 2c, d). There were more lysosomes in $786 \mathrm{O} / \mathrm{OE}-\mathrm{SR}$ cells than in $786 \mathrm{O} / \mathrm{OE}$ cells (Supplementary Fig. 2e). In 786 O/OE-SR cells, with sunitinib concentration increased, the ability of migration and invasion were enhanced (Fig. $3 f-h$ ). Rescue experiments showed that sunitinib lost its ability to enhance tumor cell metastasis without TFE3 (Fig. 3i).

\section{As a direct regulatory target of TFE3, E-Syt1 acted upstream of FAM134B during ER-phagy}

David C. Rubinsztein et al. reported that TFEB upregulated autophagy by directly binding to the CLEAR element in the promoter region of Syt $11^{23}$. We identified a homologous region of the CLEAR element in the promoter of E-Syt1, which was $-1008 \mathrm{bp}$ away from the transcription start site (TSS) (Supplementary Fig. 3a). 
a

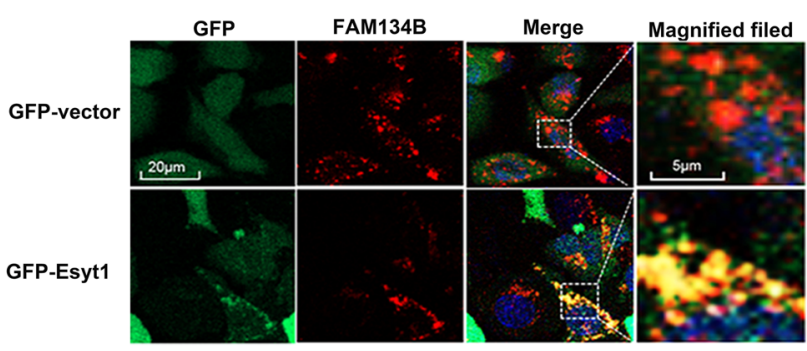

b

CCER assay
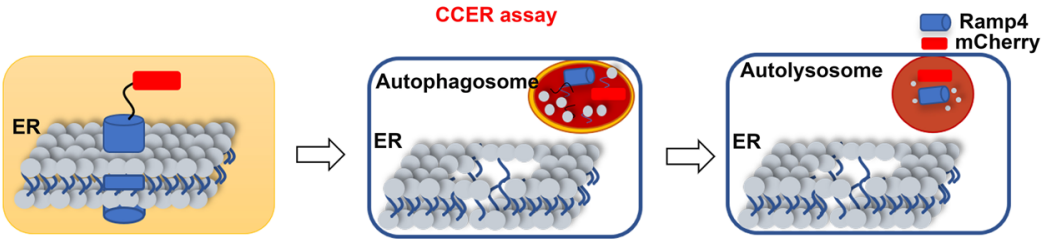

C

\section{d}

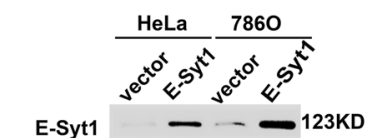

Ramp4 - - - 36KD

mCherry $-27 \mathrm{KD}$

FAM134B - - 7 70KD

LC3-I $-=-{ }_{14 \mathrm{KD}}^{16 \mathrm{KD}}$

f

GAPDH $---37 \mathrm{KD}$

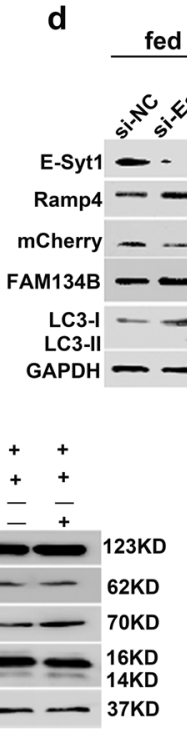

e
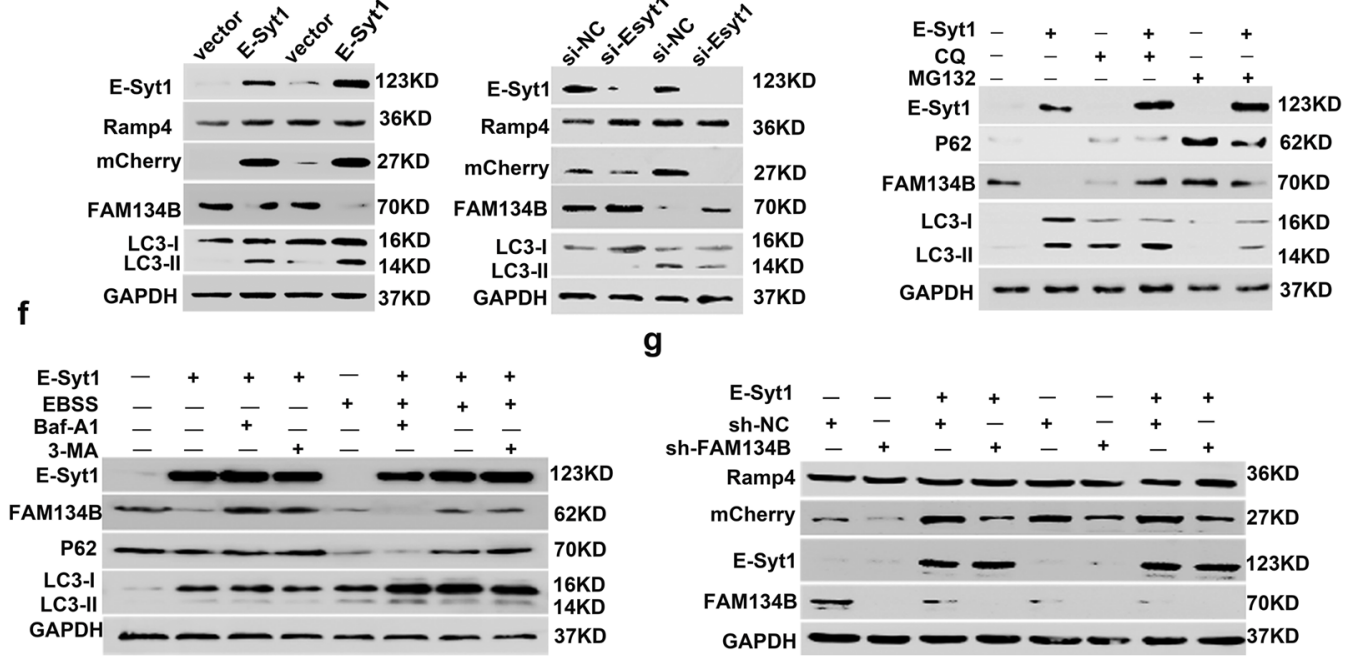

h

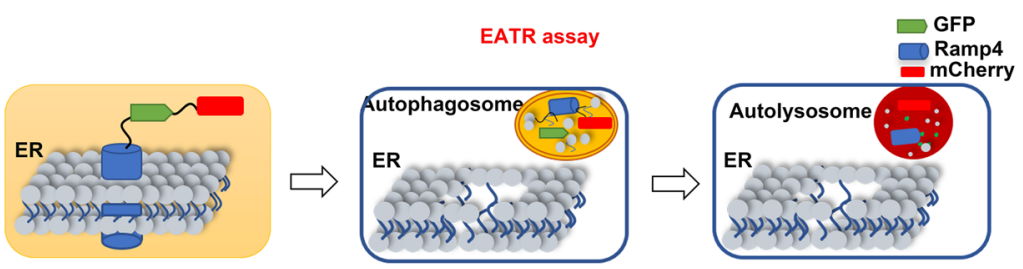

i

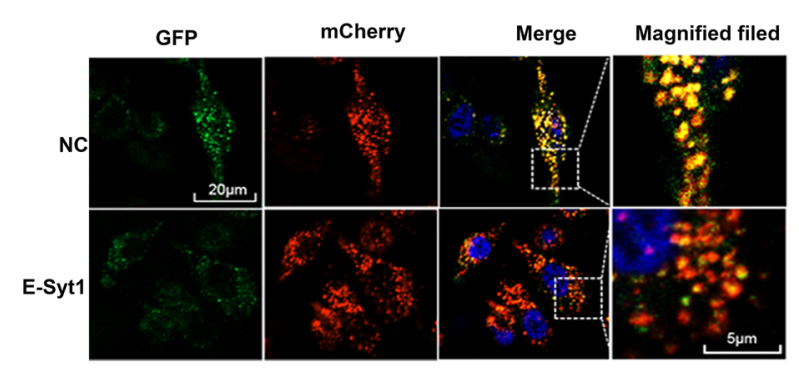

Fig. 4 (See legend on next page.) 
(see figure on previous page)

Fig. 4 TFE3 can stimulate ER-phagy by promoting the expression of E-Syt1. a Confocal images of puncta formed by Esyt1-GFP and FAM134BmCherry colocalized in 786 O/OE-SR cells. Scale bar $20 \mu \mathrm{m}$ and $5 \mu \mathrm{m}$. b Diagram of CCER assay. When ER-phagy occurs, the lysozyme cleaved the link between mCherry and RAMP4, and mCherry became a small molecule with only 27 KDa which been detected by WB. c HeLa and 786 O/OE-SR cells stably expressing CCER system were transfected with E-Syt1 and Western blot tested ER-phagy degree. $\mathbf{d} 786$ O/OE-SR cells were transfected with shEsyt1 plasmid or empty plasmid and starved for $12 \mathrm{~h}$ by EBSS before WB measurement. e Western blotting of 786 O/OE-SR cells transfected with Esyt1 and treated with MG132 $(10 \mu \mathrm{M})$ or CQ $(10 \mathrm{mM})$ for $12 \mathrm{~h}$ before harvest. $\mathrm{f} 786 \mathrm{O} / \mathrm{OE}-\mathrm{SR}$ cells transfected with E-Syt1 were either in normal condition or in EBSS with or without 3-MA (500 nM), Baf -A1 (100 nM) for $12 \mathrm{~h}$ and harvested for WB. g 786 O/OE-SR cells were transfected with E-Syt1 and shFAM134B or one of the plasmids. Then cells were cultured in normal condition or in EBSS for $12 \mathrm{~h}$. The result was shown by WB. $\mathbf{h}$ Schematic diagram of the EATR assay. When ER-phagy occurs, green fluorescence quenched in acid lysosome leaving only the red fluorescence excited by mCherry. $\mathbf{i}$ Confocal images of $786 \mathrm{O} / \mathrm{OE}-\mathrm{SR}$ cells (stabling expressing EATR system) transfected with E-Syt1 fixed to visualize ER-phagy. Scale bar $20 \mu \mathrm{m}$ and $5 \mu \mathrm{m}$.

Correlation analysis showed that E-Syt1 was positively correlated with TFE3 by RNAseq data from TCGA and GEO databases (Supplementary Fig. 3b). Our dualluciferase reporter assay proved that TFE3 could upregulate E-Syt1 by directly binding to the CLEAR element (Supplementary Fig. 3c). FAM134B and RTN3L are two key receptors of ER-phagy and one of their common interacting partners is E-Syt $1^{24}$. The co-localization of FAM134B and E-Syt1 was also observed by confocal microscopy (Fig. 4a). The distribution of E-Syt1 was different from other Esyts. E-Syt1 were widely distributed in cytoplasm, while E-Syt2 and E-Syt3 had a predominant PM-like localization ${ }^{24}$. It had reported that Esyts could recruit autophagy marker proteins at endoplasmic reticulum plasma membrane (ER-PM) contact site, thus promoting the assembly of autophagosomes ${ }^{25}$. RAMP4 is a subunit of the ER translocation complex and can be engulfed into lysosomal during ER-phagy ${ }^{22}$. We applied the mCherry cleavage from ER assay (CCER assay) to verify he regulation of ER-phagy by E-Syt1 (Fig. 4b) ${ }^{22}$. Western blotting assay showed that E-Syt1 acted upstream of FAM134B-mediated ER-phagy (Fig. 4c-g). We next used an ER autophagy tandem reporter assay (EATR assay) (Fig. 4h) to test the intensity of ER-phagy after overexpressing E-Syt1 in $786 \mathrm{O} / \mathrm{OE}-\mathrm{SR}$ cells, and the results were consistent with those before (Fig. 4i).

\section{Lysosomal releasing enzymes directly degrade the ER into fragments for autophagosome sequestration}

In order to further explore the biological function of $\mathrm{E}$ Syt1, we detected the proteins interacting with E-Syt1 in 786 O/OE-SR by mass spectrometry (MS), among which Synaptotagmin7(Syt7) attracted our interest. As a lysosomal membrane protein, Syt7 plays important role in lysosomal exocytosis, membrane resealing, and wound healing ${ }^{26}$. Analysis of the Syt7 promoter and the dualluciferase reporter assay also proved that Syt7 was downstream of TFE3 (Supplementary Fig. 4a, b). We also detected proteins that interacted with Syt7 in $786 \mathrm{O}$, $786 \mathrm{O} / \mathrm{OE}$, and $786 \mathrm{O} / \mathrm{OE}-\mathrm{SR}$ cells by MS, and the results are shown in Figure. S4c. Syt7 contains two C2 domains involving in vesicle interaction and fusion (Fig. 5a). Syt7 can form heterodimer with Esyt1 through its domain C2 domains (Fig. 5b, c). The effect of Syt7 on the degradation of Fam134B depended on the existence of E-Syt1 (Fig. 5d). After overexpressing Syt7 in 786 O/OE-SR cells, we surprisingly found that in some ER-lysosome contact sites, the ER swelled and accompanied by many ER fragments in TEM imagines (Fig. 5e). ER fragmentation is an indispensable key step of the ER-phagy and it has been reported the oligomerization of ER-phagy receptors such as FAM134B and RTN3L were the core regulators of this process $^{24,27}$. Confocal microscopy showed that the ER fragmentation induced by Syt7 extremely needed C2 domains (Fig. 5f). After siRNA silencing syt7, the diffuse distribution of CTSB decreased and the co-localization of CTSB with E-Syt1 decreased (Fig. 5g). Specific inhibition of lysosomal acid hydrolase CTSB by CA-074Me could rescue the effect of Syt7 on ER fragmentation (Fig. 5h).

\section{$\mathrm{Ca}^{2+}$ release triggered by ER-phagy promoted the lysosomal exocytosis via Syt7}

C2 domains were associated with the resistance to sunitinib in $786 \mathrm{O}$ cells (Fig. 6a). Knockdown of Syt7 temporarily by sh-RNA could reverse the resistance to sunitinib in $786 \mathrm{O} / \mathrm{OE}-\mathrm{SR}$ cells (Fig. 6b). Transwell assay showed that knocking down Syt7 could reverse the promotion of metastasis by sunitinib in $786 \mathrm{O} / \mathrm{OE}-\mathrm{SR}$ (Fig. 6c). Ionomycin is a $\mathrm{Ca}_{2}^{+}$vehicle by carrying extracellular $\mathrm{Ca}^{2+}$ into the cytoplasm so as to trigger lysosomal exocytosis. $\mathrm{C} 2$ domains function as $\mathrm{Ca}^{2+}$ sensor and can indeed trigger lysosomal exocytosis in $786 \mathrm{O} / \mathrm{OE}-\mathrm{SR}$ cells (Fig. 6d). By measuring the intracellular calcium concentration, overexpress FAM134B could directly increase intracellular $\mathrm{Ca}^{2+}$ concentration, and inhibition of CTSB can significantly reduce this process (Fig. 6e). We found that the heterodimer formed by Syt7 and E-Syt1 was essential for $\mathrm{Ca}^{2+}$ release (Fig. 6f). Sunitinib did induce lysosome exocytosis via Syt7, and this process was at least partially mediated by knockdown of E-Syt1 in our results by confocal microscopy (Fig. 6g). Because the WB results showed that the ER-phagy mediated by FAM134B could 


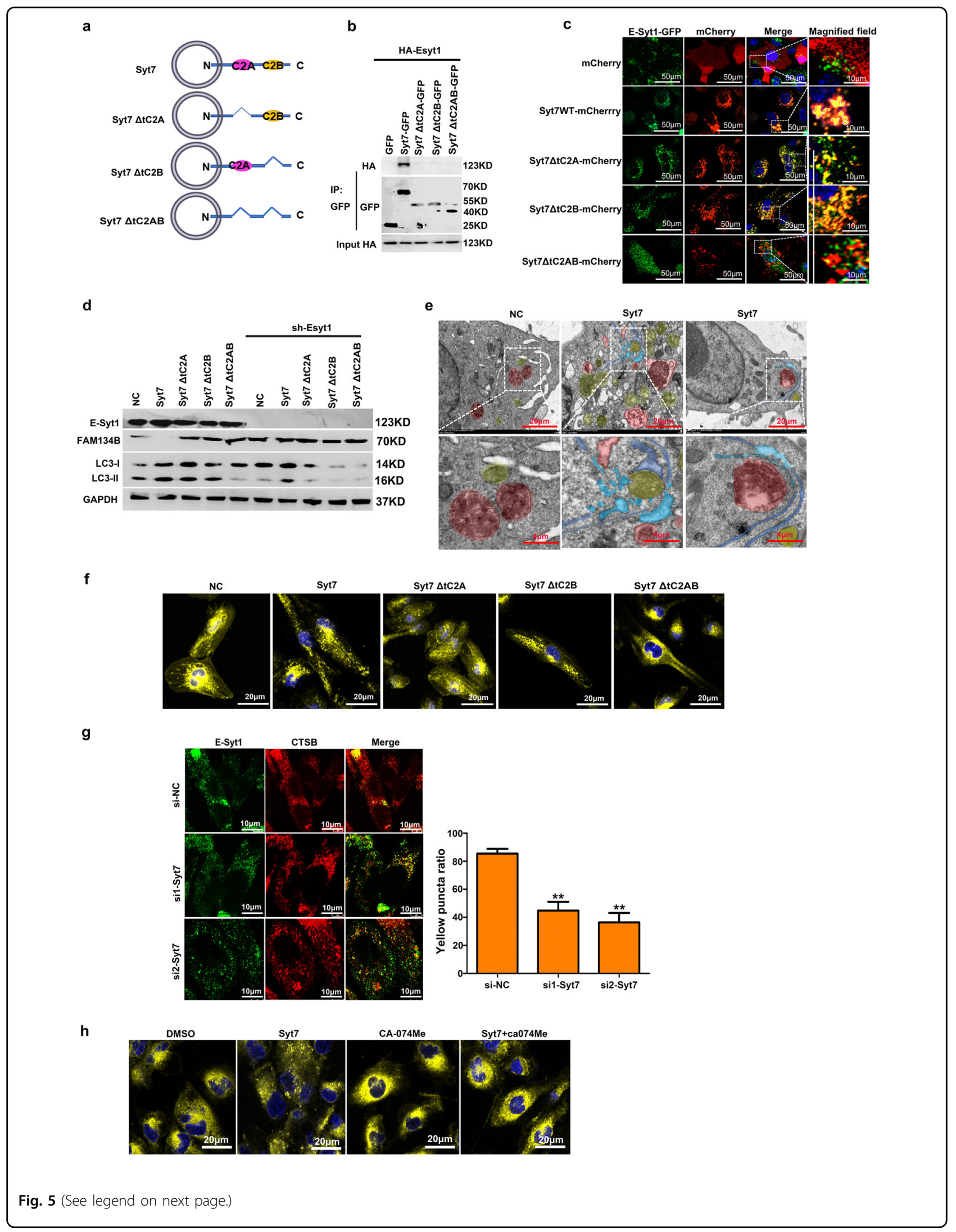


(see figure on previous page)

Fig. 5 E-Syt1/Syt7 heterodimer mediated ER fragmentation directly by lysosomal enzyme. a Domain architecture of Syt7. b Co-IP of E-Syt1 and Syt7. 293 T cells transfected with HA-Esyt1 and indicated plasmids of Syt7 mutants. Syt7 mutant proteins were immunoprecipitated using antimCherry antibody and the precipitates were analyzed using anti-HA antibody. c 786 O/OE-SR cells stably expressing GFP-Esyt1 were infected with mCherry or mCherry-tagged mutants with Syt7 by lentivirus, and nucleus stained with Hoechst 33342. Representative confocal images were shown. Scale bar $50 \mu \mathrm{m}$. Enlargement images, scale bar $10 \mu \mathrm{m}$. d Indicated plasmids of Syt7 mutants were transfected in 786 O/OE-SR cells with or without knockdown of E-Syt1. Lysates of cells were tested by WB. e 786 O/OE-SR cells transfected with Syt7 or empty vector. Representative TEM images showed that in some ER-Lysosome contact sites, ER was fragmented and engulfed by autophagosomes. Red area depicted the lysosome, yellow area indicated autophagosome, blue area indicated ER, light blue area indicated ER fragment. Scale bar $20 \mu \mathrm{m}$. Enlargement images, scale bar $4 \mu \mathrm{m}$. $\mathbf{f}$ 786 O/OE-SR cells transfected with Syt7 or mutated vector were treated with ER Tracker (yellow) and nuclear dye Hoechst 33342 (blue). ER morphology was assessed by confocal microscopy. Scale bar $20 \mu \mathrm{m}$. Scale bar $20 \mu \mathrm{m}$. g Co-localization of Esyt1 and CTSB in 786 O/OE-SR cells after siRNA mediated knockdown of Syt7. Scale bar $10 \mu \mathrm{m}$. Thirty cells were randomly selected for each treatment. The number of binding sites between E-Syt1 and CTSB in each cell was counted and the experiment was repeated 3 times (h) 786 O/OE-SR cells transfected with Syt7 or empty vector were treated with CA-074Me $(10 \mu \mathrm{M})$ or DMSO for $24 \mathrm{~h}$ and then cells were incubated with ER Tracker (yellow) and nuclear dye Hoechst 33342 (blue). ER morphology was assessed by confocal microscopy. Scale bar $20 \mu \mathrm{m}$.

reduce the degree of ER stress, we believe that the $\mathrm{Ca}^{2+}$ release was not caused by ER stress (Fig. 6h). This at least suggested that ER-phagy could alleviate ER stress in drugresistant cells. In vivo experiments also showed that knocking down Syt7 could reverse the promotion of metastasis by sunitinib in (Fig. 6i). In other words, TFE3Esyt1/Syt7 axis induced $\mathrm{Ca}^{2+}$ release by directly degrading and fragmenting ER.We draw a modle proposed on how TFE3-Esyt1/Syt7 axis regulate ER-phagy $\mathrm{Ca}^{2+}$ release and lysosomal exocytosis in Fig. 7.

\section{Discussion}

Frequent inactivation of VHL gene and activation of HIF-VEGF pathway are the main molecular biological characteristics of renal cell carcinoma, which are also the theoretical basis of antiangiogenic drug therapy. RTK inhibitors targeting VEGFR such as sunitinib and sorafenib are the first line molecular-targeted drugs for the treatment of $\mathrm{mRCC}^{28}$. Acquired resistance to RTK inhibitors and tumor metastasis are the main causes of treatment failure. However, in some tumor models, RTK inhibitor treatment increased the incidence of distant metastasis in mice ${ }^{29-34}$. Most of these reports focus on breast cancer and pancreatic cancer. Yang et al. reported that TFEB can mediate immune evasion and metastasis of RCC by inducing PD-L1 expression ${ }^{35}$. The MiT family has four major genes (TFE3, TFEB, TFEC, and MiTF). At present, most of the studies on TFE3 and cancer focus on Xp11.2 translocation renal cell carcinoma (tRCC) but not ccRCC, a special type of RCC characterized by gene fusions involving TFE3 or TFEB. Molecular signatures from proteomics gave us new insight into the metastasis mechanism of RCC after acquired sunitinib resistance. MiT family members are the most significant transcription factors by directly upregulating lysosome biosynthesis. We found that only TFE3 was upregulated in proteomic data without any other MiT family members. The VEGF/VEGFR2 pathways have three downstream: the JAK/STAT, MEK/ERK, and PI3K/AKT pathways. It has been demonstrated that 3-kinase (PI3K) is induced by VEGF/VEGFR2 can phosphorylate phosphoinositide 3kinase (PI3K) and AKT, both of which augment mammalian target of rapamycin (mTOR) activation. mTOR complex 1 (mTORC1) can respond to the nutrients level change by regulating the intracellular distribution of $\mathrm{TFE}^{20,21,36}$. In fed condition, TFE3 at serine 321 (Ser321) residue of TFE3 is phosphorylated by phosphorylated mTOR as a result of recruiting cytosolic chaperone 14-3-3 to bind to TFE3. 14-3-3 can limit the translocation of TFE3 to the nucleus ${ }^{21,37,38}$. When mTORC1 is inhibited, TFE3 will be translocated into the nucleus rapidly without the restriction of $14-3-3^{36}$. The above evidence is sufficient to indicates that sunitinib treatment can induce tumor metastasis through TFE3. Our proteomic results clearly indicated that lysosomal enzymes promote tumor metastasis by releasing them to the ECM. In other words, sunitinib has become the initiator of metastasis of innately drug-resistant RCC.

A large amount of ER-phagy was observed in metastatic tumor samples by TEM. The function of ER-phagy in cancer is unclear. Carmine settembre et al. reported that MiT/TFE factors can trigger ER-phagy by directly transcriptional regulation of FAM $134 \mathrm{~B}^{39}$. We found that ESyt1 is downstream of TFE3 and acted upstream of FAM134B during ER-phagy. The main function of E-Syt1 is ER-PM tethering ${ }^{40}$. In the initial stage of ER-phagy, FAM134B recruited LC3 to ER and meanwhile the FAM134B mediated ER fragmentation. The growing phagophores assembled by LC3 eventually engulf the ER fragments and become autophagosome ${ }^{41}$. Membrane tethering mediated FAM134B oligomers is so far the clearest mechanism of ER fragmentation ${ }^{27}$. We found that Esyt1 and Syt7 (lysosome membrane protein) could form heterodimer so as to mediates the contact between lysosome and ER and lysosomal enzymes were directly released to the nearby ER in cytoplasm, resulting in swelling and fragmentation of ER. We also observed that the ER fragmentation and nearby mature autophagosome 
a

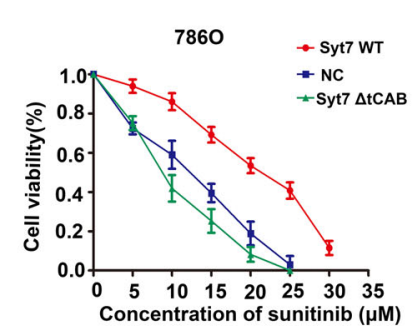

C

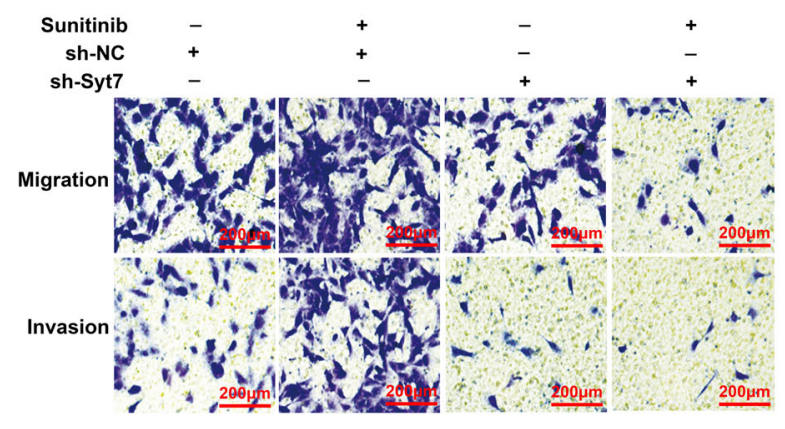

d

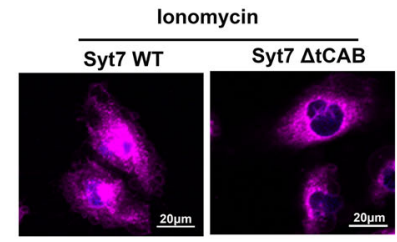

f

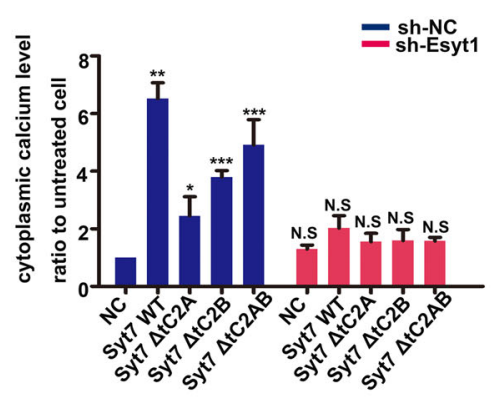

i
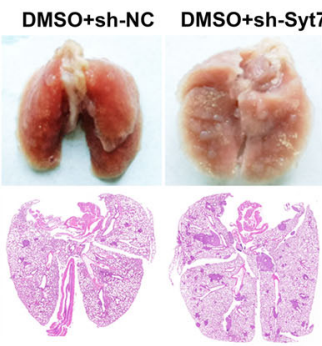

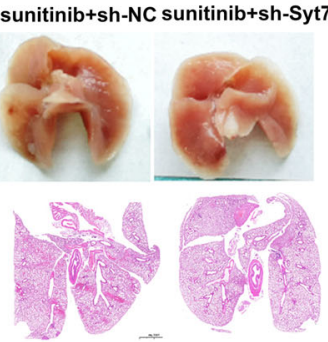

b

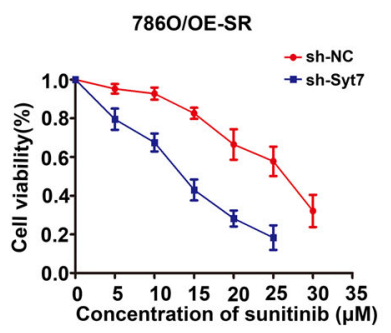

e

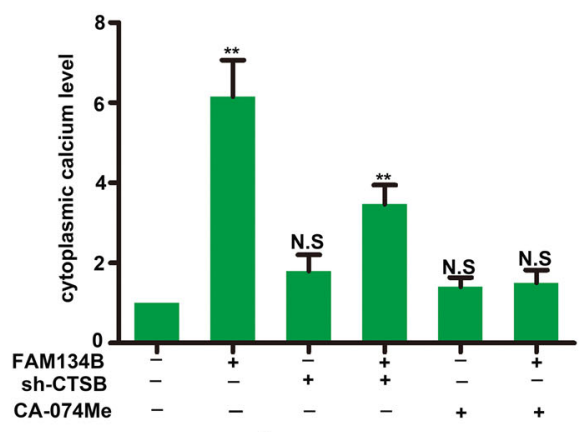

h g

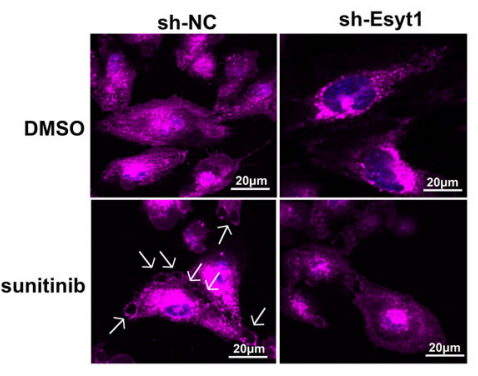

Sunitinib
FAM134B-vector

FAM134B $\quad-\longrightarrow$ 70KD

PERK - -2 140KD

P-PERK — — 140KD

IRE1 $\longrightarrow-130 \mathrm{KD}$

p-IRE1 - - 130KD

EIF2 $\alpha \longrightarrow 38 \mathrm{KD}$

p-EIF2 $\alpha-38 \mathrm{KD}$

CHOP $-27 \mathrm{KD}$ GAPDH $--37 \mathrm{KD}$

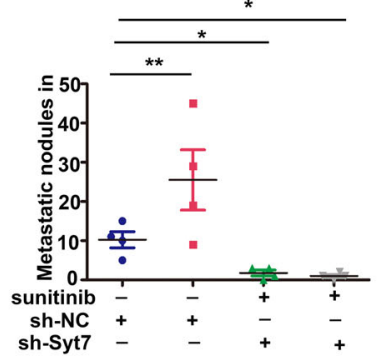

Fig. 6 (See legend on next page.) 
(see figure on previous page)

Fig. 6 ER-phagy could promote $\mathrm{Ca2}^{+}$release so as to induce Syt7 mediated lysosomal exocytosis and metastasis in 7860/OE-SR cells. a CCK8 showed the viability of $786 \mathrm{O}$ cells or transfected with Syt7, Syt7 $\triangle \mathrm{tC} 2 \mathrm{AB}$ with increasing concentration of sunitinib. Data were obtained from three independent experiments. b CCK8 showed the viability of 786 O/OE-SR cells or Syt7 knockdown 786 O/E-SR cells with increasing concentration of sunitinib. Data were obtained from three independent experiments. c Transwell assay showed that knockdown of Syt7 could reverse the effect of TFE3 on tumor metastasis in 786 O/OE-SR cells in the condition of sunitinib $(10 \mu \mathrm{M})$. Scale bar, $200 \mu \mathrm{m}{ }^{* * *} P<0.001$, ${ }^{* *} P<0.01,{ }^{*} P<0.05$. Data were obtained from three independent experiments. $\mathbf{d}$ Confocal images of vesicle exocytosis. 786 O/OE-SR cells transfected with mCherry-Syt7 or mCherry-Syt7 $\triangle \mathrm{tC} 2 \mathrm{AB}$ were incubated with ionomycin $(2 \mu \mathrm{M}) 2 \mathrm{~h}$. White arrows indicate lysosome. Scale bar $20 \mu \mathrm{m}$. e $786 \mathrm{O} / \mathrm{OE}-\mathrm{SR}$ cells transfected with FAM134B were treated with or without CA-074Me, sh-CTSB. Cells were incubated with $2 \mu \mathrm{m}$ Fluo-3-AM at for 30 min and then flow cytometry was used to test the relative $\mathrm{Ca}_{2}{ }^{+}$concentration. The results represent the mean $\pm \mathrm{SD}$. ${ }^{*} P<0.01$, NS: not significant. Data were obtained from three independent experiments. $\mathbf{f} 786 \mathrm{O} / \mathrm{OE}-\mathrm{SR}$ cells with or without E-Syt1 knockdown were transfected with indicated plasmids of Syt7 mutants. Cells were incubated with $2 \mu \mathrm{M}$ Fluo-3-AM for $30 \mathrm{~min}$ and then flow cytometry was used to test the relative $\mathrm{Ca}^{+}{ }^{+}$concentration. Results represent the mean \pm SD. ${ }^{*} P<0.05,{ }^{*} P<0.01,{ }^{* *} P<0.001$, NS: not significant. Data were obtained from three independent experiments. $\mathbf{g}$ Confocal images of vesicle exocytosis. $786 \mathrm{O} / \mathrm{OE}-\mathrm{SR}$ cells stably expressing mCherry-Syt7 were treated with DMSO or sunitinib (10 $\mu \mathrm{M})$ for $12 \mathrm{~h}$ and were transfected with sh-Esyt1 or sh-NC. White arrows indicate lysosome. Scale bar $20 \mu \mathrm{m}$. $\mathbf{h} 786 \mathrm{O} / \mathrm{OE}-\mathrm{SR}$ cells transfected with or without FAM134B-vector were incubated with sunitinib $(10 \mu \mathrm{M})$ or DMSO for $12 \mathrm{~h}$, WB tested ER stress-related markers. i Representative lungs and corresponding H\&E sections from different cohorts were shown. mice injected with Syt7 knockdown 786 O/OE-SR cells showed significantly reduced metastasis incidence in the condition of sunitinib treatment. $n=5,{ }^{*} p<0.05,{ }^{* *} p<0.01$.

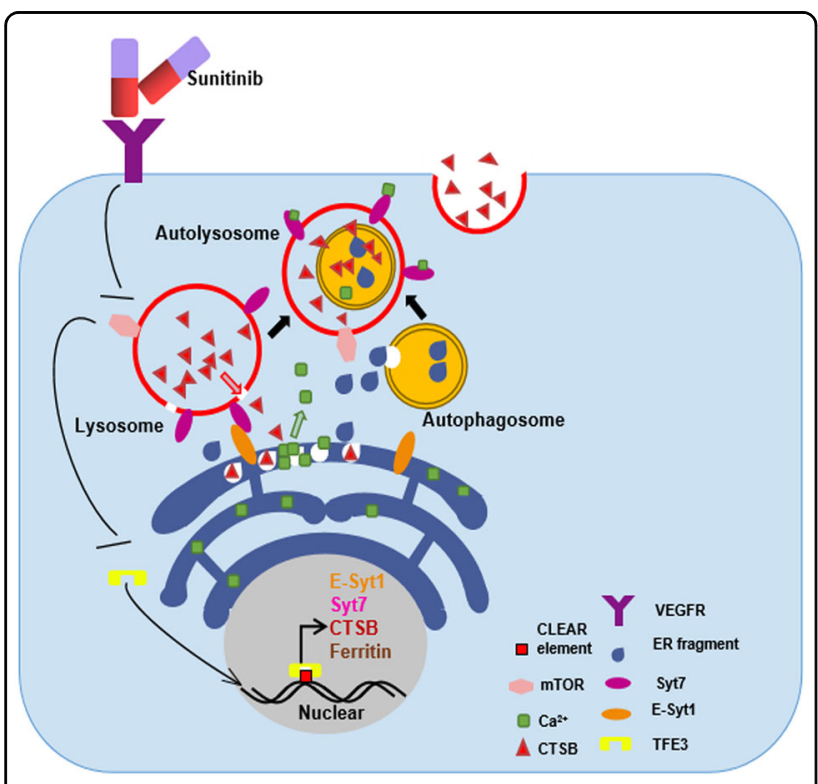

Fig. 7 The schematic illustrates the phenomena and mechanisms described in this paper. Sunitinib stimulated TFE3 to enter the nucleus, which promoted the transcription of a series of genes, and finally promoted lysosome exocytosis through ER-phagy and calcium release.

engulfing ER fragments were two relatively independent processes. We did not observe the phenomenon described in the literature: phagophore encapsulates ER fragments during growth and extension, and finally completely closed to become autophagosome ${ }^{41}$. Our findings at least provide a new perspective for the study of ER fragmentation. We further explored the relationship between ERphagy and metastasis of drug-resistant tumors cells and found that Syt7 was a very important linker between these biological phenomena. ER fragmentation mediated by heterodimerization between Syt7 and E-Syt1 resulted in the releasing of calcium ions from ER to the cytoplasm. As a calcium sensor on lysosomal membrane, Syt7 can sense the increase of calcium ion concentration in the cytoplasm and trigger lysosome exocytosis. We speculate that exocytosis of lysosome can not only eliminate the isolated sunitinib in lysosome, but also degrade the ECM to promote metastasis of tumor cells.

There is a kind of clinical subtype of RCC which will develop rapid disease progression after TKI therapy ${ }^{42}$, the case we studied is not of this type. The patient received a satisfactory progression-free survival (PFS) by sunitinib treatment and experienced obvious tumor shrinkage. Sunitinib is still the best choice for this case. Therefore, it is urgent to monitor the resistance of sunitinib in RCC during treatment. Based on proteomic data. Our proteomics results also confirmed Marija Petkovic's hypotheses that mTOR pathway inhibitors will also result in changes in iron and ferritin level in $\mathrm{mRCC}^{42}$. The relationship between TFE3 and ferritin needs further exploration.

TFE3 is highly expressed in RCC tissue. Although the expression of TFE3 or TFEB in RCC has no statistical significance for prognosis, it does not mean that there is no significance under the effect of targeted drugs. This study suggests that it is necessary to monitor the sensitivity of the tumor to sunitinib in some mRCC patients with high expression of TFE3 or TFEB.

\section{Acknowledgements}

We thank Yang Jiang for technical assistance and virus packaging.

\section{Funding statement}

The study was funded by the National Natural Science Foundation of China (Nos.: 81372765, 81572515, 81711530048, 81472395, 81672522); Shandong Provincial Natural Science Foundation, China (Nos.: ZR2011HM055, ZR2014HQ035, BS2014YY036); Research fund of Shandong University Qilu Hospital (2014QLKY16). 


\section{Author contributions}

Y.D.F., C.L., J.K.L., and L.C.L. designed the paper. L.C.L., S.Z., Z.F.L., and S.P. performed and analyzed experiments and wrote the paper. N.Z.Z. and L.C.L. analyzed data and reviewed the paper. Y.D.F., L.C.L. designed, supervised, and analyzed experimental work and wrote the paper.

\section{Ethics statement}

All animal work procedures were approved by the Ethics Committee of the Shandong University Qilu Hospital.

\section{Conflict of interest}

The authors declare no competing interests.

\section{Publisher's note}

Springer Nature remains neutral with regard to jurisdictional claims in published maps and institutional affiliations.

Supplementary information The online version contains supplementary material available at https://doi.org/10.1038/s41419-021-03511-3.

Received: 6 November 2020 Revised: 21 January 2021 Accepted: 26 January 2021

Published online: 26 February 2021

\section{References}

1. Parkin, D. M., Bray, F., Ferlay, J. \& Pisani, P. Global cancer statistics, 2002. Cancer J. Clin. 55, 74-108 (2005).

2. Rini, B. I., Campbell, S. C. \& Escudier, B. Renal cell carcinoma. Lancet 373 1119-1132 (2009).

3. Gossage, L., Eisen, T. \& Maher, E. R. VHL, the story of a tumour suppressor gene. Nat. Rev. Cancer 15, 55-64 (2015).

4. Gossage, L. \& Eisen, T. Alterations in VHL as potential biomarkers in renal-cell carcinoma. Nat. Rev. Clin. Oncol. 7, 277-288 (2010).

5. Linehan, W. M., Srinivasan, R. \& Schmidt, L. S. The genetic basis of kidney cancer: a metabolic disease. Nat. Rev. Urol. 7, 277-285 (2010).

6. Huang, Q. et al. Androgen receptor increases hematogenous metastasis yet decreases lymphatic metastasis of renal cell carcinoma. Nat. Commun. 8, 918 (2017).

7. Xue, D. et al. Circ-AKT3 inhibits clear cell renal cell carcinoma metastasis via altering miR-296-3p/E-cadherin signals. Mol. Cancer 18, 151 (2019).

8. Ni, D. et al. Downregulation of FOXO3a promotes tumor metastasis and is associated with metastasis-free survival of patients with clear cell renal cell carcinoma. Clin. Cancer Res. 20, 1078-0432 (2014).

9. Kolter, T. \& Sandhoff, K. Lysosomal degradation of membrane lipids. FEBS Lett. 584, 1700-1712 (2010).

10. Schulze, H., Kolter, T. \& Sandhoff, K. Principles of lysosomal membrane degradation: cellular topology and biochemistry of lysosomal lipid degradation. Biochim. Biophys. Acta 1793, 674-683 (2009).

11. Pryor, P. R. \& Luzio, J. P. Delivery of endocytosed membrane proteins to the lysosome. Biochim. Biophys. Acta 1793, 615-624 (2009).

12. Saftig, P. \& Klumperman, J. Lysosome biogenesis and lysosomal membrane proteins: trafficking meets function. Nat. Rev. Mol. Cell Biol. 10, 623-635 (2009).

13. Khaket, T. P., Kwon, T. K. \& Kang, S. C. Cathepsins: potent regulators in carcinogenesis. Pharmacol. Therapeutics 198, 1-19 (2019).

14. Padamsey, Z. et al. Activity-dependent exocytosis of lysosomes regulates the structural plasticity of dendritic spines. Neuron 93, 132-146 (2017).

15. Olson, O. C. \& Joyce, J. A. Cysteine cathepsin proteases: regulators of cancer progression and therapeutic response. Nat. Rev. Cancer 15, $712-729$ (2015).

16. Giuliano, S. et al. Resistance to sunitinib in renal clear cell carcinoma results from sequestration in lysosomes and inhibition of the autophagic flux. Autophagy 11, 1891-1904 (2015).

17. Zhitomirsky, B. \& Assaraf, Y. G. Lysosomes as mediators of drug resistance in cancer. Drug Resistance Updates: Rev. Commentaries Antimicrobial Anticancer Chemother. 24, 23-33 (2016).
18. Gotink, K. J. et al. Lysosomal sequestration of sunitinib: a novel mechanism of drug resistance. Clin. Cancer Res.: Off. J. Am. Assoc. Cancer Res.17, 7337-7346 (2011).

19. Sardiello, M. et al. A gene network regulating lysosomal biogenesis and function. Science 325, 473-477 (2009).

20. Settembre, C. et al. TFEB links autophagy to lysosomal biogenesis. Science $\mathbf{3 3 2}$, 1429-1433 (2011)

21. Martina, J. A. et al. The nutrient-responsive transcription factor TFE3 promotes autophagy, lysosomal biogenesis, and clearance of cellular debris. Sci. Signal. 7, ra9 (2014).

22. Liang, J. R., Lingeman, E., Ahmed, S. \& Corn, J. E. Atlastins remodel the endoplasmic reticulum for selective autophagy. J. Cell Biol. 217, 3354-3367 (2018).

23. Bento, C. F., Ashkenazi, A., Jimenez-Sanchez, M. \& Rubinsztein, D. C. The Parkinson's disease-associated genes ATP13A2 and SYT11 regulate autophagy via a common pathway. Nat. Commun. 7, 11803 (2016).

24. Grumati, P. et al. Full length RTN3 regulates turnover of tubular endoplasmic reticulum via selective autophagy. elife 6, https://doi.org/10.7554/eLife.25555 (2017).

25. Nascimbeni, A. C. et al. ER-plasma membrane contact sites contribute to autophagosome biogenesis by regulation of local PI3P synthesis. EMBO J. $\mathbf{3 6}$ 2018-2033 (2017).

26. Colvin, R. A. et al. Synaptotagmin-mediated vesicle fusion regulates cell migration. Nat. Immunol. 11, 495-502 (2010).

27. Jiang, $X$. et al. FAM134B oligomerization drives endoplasmic reticulum membrane scission for ER-phagy. EMBO J. 39, e102608 (2020).

28. Rixe, O. et al. A randomized, phase II, dose-finding study of the pan-ErbB receptor tyrosine-kinase inhibitor $\mathrm{Cl}-1033$ in patients with pretreated metastatic breast cancer. Cancer Chemother. Pharmacol. 64, 1139-1148 (2009).

29. Cooke, V. G. et al. Pericyte depletion results in hypoxia-associated epithelial-tomesenchymal transition and metastasis mediated by met signaling pathway. Cancer Cell 21, 66-81 (2012).

30. Pàez-Ribes, M. et al. Antiangiogenic therapy elicits malignant progression of tumors to increased local invasion and distant metastasis. Cancer Cell 15, 220-231 (2009).

31. Sennino, B. et al. Suppression of tumor invasion and metastasis by concurrent inhibition of c-Met and VEGF signaling in pancreatic neuroendocrine tumors. Cancer Discov. 2, 270-287 (2012).

32. Yin, T. et al. Antiangiogenic therapy using sunitinib combined with rapamycin retards tumor growth but promotes metastasis. Transl. Oncol. 7, 221-229 (2014).

33. Welti, J. C. et al. Contrasting effects of sunitinib within in vivo models of metastasis. Angiogenesis 15, 623-641 (2012).

34. Wragg, J. W., Heath, V. L. \& Bicknell, R. Sunitinib treatment enhances metastasis of innately drug-resistant breast tumors. Cancer Res. 77. 1008-1020 (2017).

35. Zhang, C. et al. TFEB mediates immune evasion and resistance to mTOR inhibition of renal cell carcinoma via induction of PD-L1. Clin. Cancer Res.: Off. J. Am. Assoc. Cancer Res. 25, 6827-6838 (2019).

36. Yin, X. et al. TFE3 fusions escape from controlling of mTOR signaling pathway and accumulate in the nucleus promoting genes expression in Xp11.2 translocation renal cell carcinomas. J. Exp. Clin. Cancer Res. 38, 119 (2019).

37. Roczniak-Ferguson, A. et al. The transcription factor TFEB links mTORC1 signaling to transcriptional control of lysosome homeostasis. Sci. Signal. 5, ra42 (2012).

38. Settembre, C. et al. A lysosome-to-nucleus signalling mechanism senses and regulates the lysosome via mTOR and TFEB. EMBO J. 31, 1095-1108 (2012).

39. Cinque, L. et al. MiT/TFE factors control ER-phagy via transcriptional regulation of FAM134B. EMBO J. 39, e105696 (2020).

40. Giordano, F. et al. PI(4,5)P(2)-dependent and $\mathrm{Ca}\left(2^{+}\right)$-regulated ER-PM interactions mediated by the extended synaptotagmins. Cell 153, 1494-1509 (2013).

41. Popelka, H. \& Klionsky, D. J. Molecular dynamics simulations reveal how the reticulon-homology domain of the autophagy receptor RETREG1/FAM134B remodels membranes for efficient selective reticulophagy. Autophagy 16, 585-588 (2020).

42. Bersanelli, M. et al. Metastatic renal cell carcinoma rapidly progressive to sunitinib: what to do next? Eur. Urol. Oncol. https://doi.org/10.1016/j. euo.2019.06.018 (2019). 